\title{
Wind-Wave Misalignment Effects on Langmuir Turbulence in Tropical Cyclone Conditions
}

\author{
DONG WANG AND TOBIAS KUKULKA \\ University of Delaware, Newark, Delaware \\ BRANDON G. REICHL \\ Program in Atmospheric and Oceanic Sciences, Princeton University, Princeton, New Jersey \\ TETSU HARA AND ISAAC GINIS \\ University of Rhode Island, Narragansett, Rhode Island
}

(Manuscript received 18 April 2019, in final form 20 September 2019)

\begin{abstract}
This study utilizes a large-eddy simulation (LES) approach to systematically assess the directional variability of wave-driven Langmuir turbulence (LT) in the ocean surface boundary layer (OSBL) under tropical cyclones (TCs). The Stokes drift vector, which drives LT through the Craik-Leibovich vortex force, is obtained through spectral wave simulations. LT's direction is identified by horizontally elongated turbulent structures and objectively determined from horizontal autocorrelations of vertical velocities. In spite of a TC's complex forcing with great wind and wave misalignments, this study finds that LT is approximately aligned with the wind. This is because the Reynolds stress and the depth-averaged Lagrangian shear (Eulerian plus Stokes drift shear) that are key in determining the LT intensity (determined by normalized depth-averaged vertical velocity variances) and direction are also approximately aligned with the wind relatively close to the surface. A scaling analysis of the momentum budget suggests that the Reynolds stress is approximately constant over a near-surface layer with predominant production of turbulent kinetic energy by Stokes drift shear, which is confirmed from the LES results. In this layer, Stokes drift shear, which dominates the Lagrangian shear, is aligned with the wind because of relatively short, wind-driven waves. On the contrary, Stokes drift exhibits considerable amount of misalignments with the wind. This wind-wave misalignment reduces LT intensity, consistent with a simple turbulent kinetic energy model. Our analysis shows that both the Reynolds stress and LT are aligned with the wind for different reasons: the former is dictated by the momentum budget, while the latter is controlled by wind-forced waves.
\end{abstract}

\section{Introduction}

The interaction of wave-induced Stokes drift and the vorticity of turbulent currents results in the CraikLeibovich (CL) vortex force, which drives an oceanic turbulent process called Langmuir turbulence (LT) (Craik and Leibovich 1976). Recent studies show that LT plays an important role in the upper-ocean mixing under tropical cyclones (TCs) (Sullivan et al. 2012; Rabe et al. 2015; Reichl et al. 2016a,b; Wang et al. 2018). For instance, LT enhances turbulent entrainment, resulting in greater mixed layer deepening and sea surface cooling, which critically affects the air-sea heat fluxes that

\footnotetext{
Corresponding author: Dong Wang, dongwang@udel.edu
}

drive the development of the TC. Most TCs feature extreme, transient winds, which generate nonequilibrium waves with widely distributed wind-wave misalignments (Fan et al. 2009). The wind-wave misalignments are found to reduce LT intensity and change the orientation of the Langmuir cell (LC), which illustrates the important role of the wind-wave misalignment in LT's development and parameterizations (Van Roekel et al. 2012; Sullivan et al. 2012; Rabe et al. 2015).

LT intensity is often characterized by the depthaveraged vertical velocity variance (VVV), which is generally small in the purely shear-driven turbulence (ST) but can be significantly increased by LT-enhanced vertical turbulent transport (Polton and Belcher 2007; Li et al. 2005; Harcourt and D'Asaro 2008). Rabe et al. (2015) 
shows that the great wind-wave misalignment under the TC can reduce the enhancement of VVV by LT toward levels of ST alone. It has been shown that wind-wave misalignment contributes to vorticity transport across Langmuir cells, thereby reducing LT (Van Roekel et al. 2012). Wind-wave misalignment also decreases the production of turbulent kinetic energy (TKE) due to waves (Van Roekel et al. 2012; Sullivan et al. 2012; Rabe et al. 2015). The TKE production due to nonbreaking surface waves is mathematically represented by the dot product of the Reynolds stress vector and Stokes drift shear vector (McWilliams et al. 1997; Grant and Belcher 2009). Thus, the profile of Reynolds stress is another important factor that critically influences LT intensity and structure in addition to the Stokes drift shear that is imposed as a wave forcing.

For steady flow, the Reynolds stress profile in the ocean surface boundary layer (OSBL) is often described by an Ekman balance between the Coriolis force and divergences of the turbulent Reynolds stresses. This balance leads to downward spiraling and decaying current structure (Price et al. 1987; Polton et al. 2013). However, in TC conditions, the forcing is highly transient, so that deviations from steady-state dynamics are expected (Sullivan et al. 2012). Furthermore, LT-enhanced mixing and the interaction of Stokes drift and Coriolis force causes a reduced decay of the Ekman spiral with depth (McWilliams and Sullivan 2000). With LT, the direction of Reynolds stress is found to be more aligned with the Lagrangian current shear than the mean Eulerian current shear (McWilliams and Sullivan 2000; McWilliams et al. 2012; Reichl et al. 2016b). Note that the Lagrangian current is the sum of Eulerian current and Stokes drift vectors. The relationship between current shear and Reynolds stress is critical for Reynolds stress parameterizations. It is traditionally assumed that the direction and magnitude of the Reynolds stress is directly related to the Eulerian shear (Pope 2010).

In addition to reducing LT intensity, the wind-wave misalignment also impacts LT direction. The direction of LT is a crucial parameter in quantifying the effects of wind-wave misalignment on LT intensity since only the TKE and vorticity productions along LT direction contribute to the growth of LT (Van Roekel et al. 2012; Leibovich 1983). LT direction is often identified by its horizontally elongated turbulent structures, which are generated by strong downwelling flows (Kukulka et al. 2010; Van Roekel et al. 2012; Sullivan et al. 2012). For moderate winds, LT direction changes with wind-wave misalignment and is approximately between the wind and wave directions (Van Roekel et al. 2012). Theoretical studies indicate that LT is directed to maximize the wave-current interaction and minimize the cross-LC shear. This requires the LT direction to be along the Lagrangian current shear, which also controls the generation of the along-LC vorticity (Gnanadesikan and Weller 1995; Holm 1996). Thus, the Lagrangian shear is the key factor that determines LT direction, which is a function of depth.

In TC conditions, LT direction has been examined at the depth of maximum vertical velocity variance and compared to the direction of the Lagrangian shear at the same depth. This comparison suggests these directions are nearly aligned, although the full three-dimensional LT structure for a wide range of conditions has not yet been investigated (Sullivan et al. 2012). In fact, previous studies identify LT direction at a single depth layer, ignoring any depth variation of LT direction that has been observed in extreme swell conditions (McWilliams et al. 2014). This also motivates a critical examination of using a depth-averaged Lagrangian shear to predict LT direction (Van Roekel et al. 2012). Furthermore, Sullivan et al. (2012) shows that LT direction is approximately aligned with the wind direction in spite of TC's substantial wind-wave misalignments. This result stands in contrast with the early finding that LT direction is between the wind and wave directions.

TC winds drive breaking waves, which substantially affect turbulence dynamics in a relatively thin near-surface layer that is much smaller than the ocean surface boundary layer and characterized by a predominant balance of TKE transport and dissipation (Craig and Banner 1994; Terray et al. 1996; Noh et al. 2004; Sullivan et al. 2007). The breaking wave affected layer is often assumed to scale with significant wave height, which is much smaller than the ocean surface boundary layer (OSBL) depth and, thus, usually assumed to not greatly impact the dynamics of the deeper OSBL, which is in agreement with previous LES studies with explicit breaking wave effects (Noh et al. 2004; Sullivan et al. 2007; Kukulka and Brunner 2015). Consistent with our previous studies (Reichl et al. 2016b; Wang et al. 2018) and other LES studies under TC (e.g., Sullivan et al. 2012; Rabe et al. 2015), we do not explicitly include breaking waves in our LES approach.

This study investigates the influence of wind-wave misalignment on LT intensity and direction for a wide range of TC conditions. Our results play a key role in understanding misalignment effects in complex forcing conditions, scaling LT, and for developing comprehensive turbulence closure schemes of the OSBL. In section 2 , we briefly review the methods in our study, which follows the same numerical approaches as Reichl et al. (2016a,b) and Wang et al. (2018). In the results section, we first examine important factors that control LT intensity and direction (sections $3 \mathrm{a}-\mathrm{c}$ ) and then assess the 
effects of the wind-wave misalignment on LT, illustrating that TC's wind-wave misalignment reduces LT intensity (section $3 \mathrm{~d}$ ) but does not affect LT direction (section 3e).

\section{A synopsis of OSBL turbulent models}

\section{a. Wind and wave models}

This study is a follow-up study of our previous investigations on the response of LT to TCs. We use the same wind and wave models and setups as in our previous studies (Reichl et al. 2016a,b; Wang et al. 2018). The wind field is prescribed from the TC-tuned Holland wind model (Holland 1980, 2008). This wind is used as the input for the third-generation wave model WAVEWATCH III (Tolman 2009) to simulate the wave field. The TC wind translation speed is set to $5 \mathrm{~m} \mathrm{~s}^{-1}$ moving from east to west, the maximum wind speed is set to $65 \mathrm{~m} \mathrm{~s}^{-1}$ (at $10-\mathrm{m}$ height), and the radius of maximum wind (RMW) is set to $50 \mathrm{~km}$.

The output of the wind model is the wind vector at 10 - $\mathrm{m}$ height $\mathbf{U}_{10}$ and for the wave model it is the twodimensional wave height spectrum $F(\omega, \theta)$ where $\omega$ is radian frequency and $\theta$ is the wave propagation direction. The wind and wave forcing that we use to drive the OSBL model are the wind stress vector $\tau_{0}$ and Stokes drift vector $\mathbf{u}_{s}$, respectively. The wind stress vector $\boldsymbol{\tau}_{0}$ is parameterized through $\boldsymbol{\tau}_{0}=C_{d} \rho_{a}\left|\mathbf{U}_{10}\right| \mathbf{U}_{10}$, where $\rho_{a}$ is the air density and $C_{d}$ is the drag coefficient depending on $\left|\mathbf{U}_{10}\right|$ (Sullivan et al. 2012). The wind stress vector $\boldsymbol{\tau}_{0}$ is also used to obtain the water friction velocity $u_{*}$ in the OSBL model following $\left|\boldsymbol{\tau}_{0}\right|=\rho_{0} u_{*}^{2}$, where $\rho_{0}=1024 \mathrm{~kg} \mathrm{~m}^{-3}$ is the reference density. The depth-dependent $\mathbf{u}_{s}$ is obtained by integrating the wave spectra following:

$$
\mathbf{u}_{s}(z)=2 \int_{0}^{\infty} \int_{-\pi}^{\pi} \mathbf{k} \omega F(\omega, \theta) e^{2|\mathbf{k}| z} d \theta d \omega,
$$

where $\mathbf{k}$ is the horizontal wavenumber vector and $z$ is the vertical coordinate $(z=0$ at the mean sea surface and $z<0$ in the ocean) (Kenyon 1969). Other details of the computational parameters and setups of the wind and wave models are described in Wang et al.'s (2018) study. In TC conditions, $\mathbf{u}_{s}$ at $z=-5 \mathrm{~m}$ varies spatiotemporally due to the transient wind forcing (Fig. 1). For instance, the wave forcing is generally stronger on the right of the TC track (Northern Hemisphere) because waves propagate with the TC and experience greater wind forcing, creating more favorable conditions for wave's growth. In addition, the wind-wave misalignment is ubiquitous under the $\mathrm{TC}$ and can be more than $90^{\circ}$ in some regions, particularly on the left of the TC track (misalignment is illustrated by the angle between the black and gray

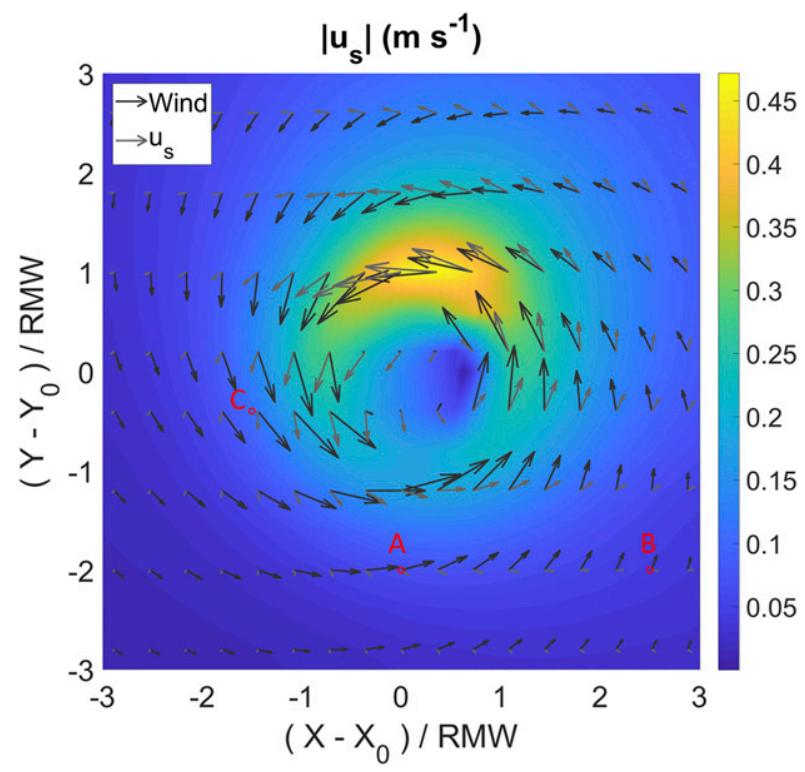

FIG. 1. The magnitude of Stokes drift at $z=-5 \mathrm{~m}$ over the TC domain. The directions of wind and $\mathbf{u}_{s}$ are indicated by black and gray arrows at select locations. The longest black and gray arrows correspond to the maximum wind speed of $65 \mathrm{~m} \mathrm{~s}^{-1}$ and the maximum $\left|\mathbf{u}_{s}\right|$ of $0.5 \mathrm{~m} \mathrm{~s}^{-1}$, respectively. The red circles marked by the capital letters $\mathrm{A}, \mathrm{B}$, and $\mathrm{C}$ indicate the three locations that are examined in section 3. Note that the $x$ axis represents the translating distance of the TC relatively to where the TC's eye passes $\left(X_{0}\right)$, and the $y$ axis denotes the distance of each station from the TC's eye $\left(Y_{0}\right)$. Both distances are normalized by the radius of the maximum wind (RMW), which is $50 \mathrm{~km}$.

arrows, Fig. 1), which plays an important role in influencing LT's development.

\section{b. Ocean surface boundary layer model}

The upper-ocean response to the TC forcing is simulated by a large-eddy simulation (LES) model based on the grid-filtered CL equations (Craik and Leibovich 1976; McWilliams et al. 1997; Skyllingstad and Denbo 1995; Kukulka et al. 2010). LT is generated by a vortex force in the CL equations, that is the cross product of the Stokes drift $\mathbf{u}_{s}$ and vorticity. When there are no waves $\left(\mathbf{u}_{s}=0\right)$, this vortex force is zero and the grid-filtered CL equations are identical to the grid-filtered Navier-Stokes equations that only simulate shear-driven turbulence (ST). In this study, we use the same LES experiments and setups as in Reichl et al. (2016b) and Wang et al. (2018), including simulations with and without LT at 18 stations along the $Y$ direction, which is perpendicular to the TC's propagation direction $X$ (Fig. 6). The range of $Y$ explored here is from -200 to $200 \mathrm{~km}$, where $Y_{0}=0$ is at the TC's center and $Y>0$ and $Y<0$ denote stations on the right and left of the TC's eye, respectively. Each simulation has a physical duration time $t=72 \mathrm{~h}$, and the 
TABLE 1. Locations of nine stations with outputs of 3D flow field.

\begin{tabular}{cccccccccc}
\hline \hline Station number & 19 & 20 & 21 & 22 & 23 & 24 & 25 & 26 & 27 \\
\hline$Y-Y_{0}(\mathrm{~km})$ & 150 & 80 & 30 & 10 & 0 & -20 & -70 & -80 & -150 \\
\hline
\end{tabular}

TC eye passes each station at $t_{0}=36 \mathrm{~h}$. Since the forcing and response are in quasi-steady state with respect to the TC translation, we can transform the evolution time into moving distance using $X=V\left(t-t_{0}\right)$, where $V$ is the translation speed (here $5 \mathrm{~m} \mathrm{~s}^{-1}$ ). Therefore, $X<0$ and $X>0$ correspond to time periods before and after the TC eye passes, respectively, with $X_{0}=0$ corresponding to $t_{0}$.

In addition to the simulations presented in Reichl et al. (2016b) and Wang et al. (2018), we conduct another set of LES experiments at a subset of stations ranging from -150 to $150 \mathrm{~km}$ along transect $Y$, perpendicular to the storm translation direction, to examine in detail the spatial structure of LT (Table 1). For these experiments, the LES domain is $300 \mathrm{~m} \times 300 \mathrm{~m}$ wide and $250 \mathrm{~m}$ deep with $228 \times 228$ horizontal grid points and 256 vertical grid points. The horizontal spatial coordinate in the LES domain is denoted by $(x, y)$, of which positive directions are along east and north, respectively. Consistent with Wang et al. (2018), we prescribe a horizontally uniform initial temperature profile, characterized by a 10-m mixed layer depth with a uniform temperature $T=$ $302.4 \mathrm{~K}$ and a constant stratification of $d T / d z=0.04 \mathrm{~K} \mathrm{~m}^{-1}$ below. A constant heat flux of $-5 \mathrm{~W} \mathrm{~m}^{-2}$ is imposed to accelerate the initial spinup of turbulence. Along with the frequently saved [per $\mathscr{O}\left(10^{2}\right) \mathrm{s}$ ] horizontally averaged profiles of simulation results, fully three-dimensional turbulence fields are saved hourly. Sensitivity tests indicate that the smaller horizontal and vertical domain sizes and higher resolution does not significantly influence our results but high resolution enhances the visual representation of flow fields.

\section{Results}

To understand the impact of wind-wave misalignment on LT intensity and direction, we first examine the profiles of Reynolds stress $(\boldsymbol{\tau})$, Stokes drift shear $\left(\partial \mathbf{u}_{s} / \partial z\right)$, and Lagrangian shear $\left[\partial\left(\mathbf{u}_{s}+\mathbf{u}\right) / \partial z\right]$. These quantities combine to control LT production and determine LT direction (sections $3 a-c)$. We then assess the wind-wave misalignment effects in TC conditions on LT intensity (section 3d) and direction (section 3e) and discuss how wind-wave misalignment under complex TC conditions affects LT intensity and LT direction.

\section{a. Layer with substantial stress}

We first examine $\tau$ at the depth $z=-0.2 H_{B}$ to estimate what happens to the profile down to a greater depth relative to the surface, where $H_{B}$ is a boundary layer depth (defined to be the depth where the turbulent stress decays to $5 \%$ of its surface value). The Reynolds stress $\tau$ is aligned with the wind in most of the TC domain except regions near the TC eye, where the wind decays and turns rapidly (left panel, Fig. 2 and Fig. 1). In addition, the magnitude of $\tau$ decays little within $0.2 H_{B}$
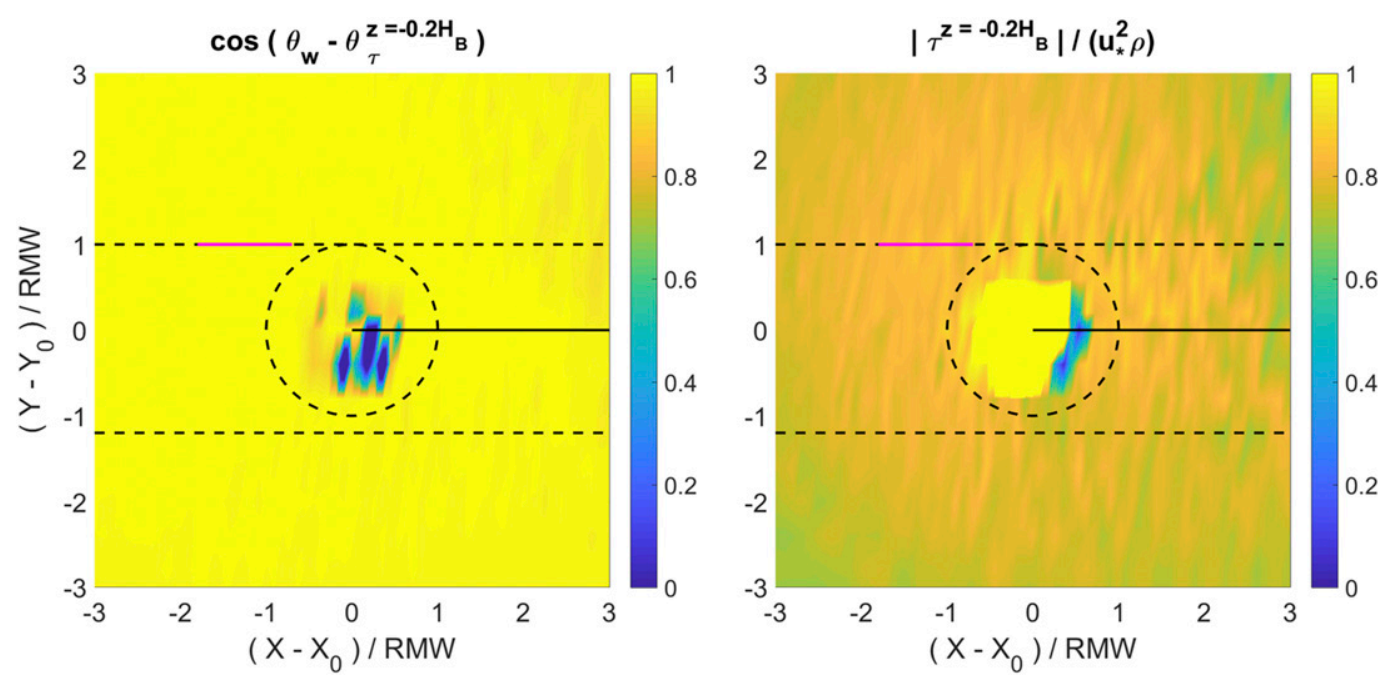

FIG. 2. (left) The misalignment between the wind direction $\theta_{w}$ and the direction of Reynolds stress $\theta_{\tau}$ at depth $z=$ $-0.2 H_{B}$. (right) The normalized Reynolds stress by $u_{*}^{2} \rho$ at $z=-0.2 H_{B}$. In the right panel, the normalized Reynolds stress exceeds one inside the RMW, where the color is saturated. The two horizontal dashed black lines denote two LES transects at $\left(Y-Y_{0}\right) / \mathrm{RMW}=1$ and $\left(Y-Y_{0}\right) / \mathrm{RMW}=-1.2$, and the dashed black circle indicates the radius of maximum wind. The solid black line is the path of the TC moving from right to left. The solid magenta line denotes the range of Reynolds stress and mean current shear profiles that will be examined in Fig. 5 . 

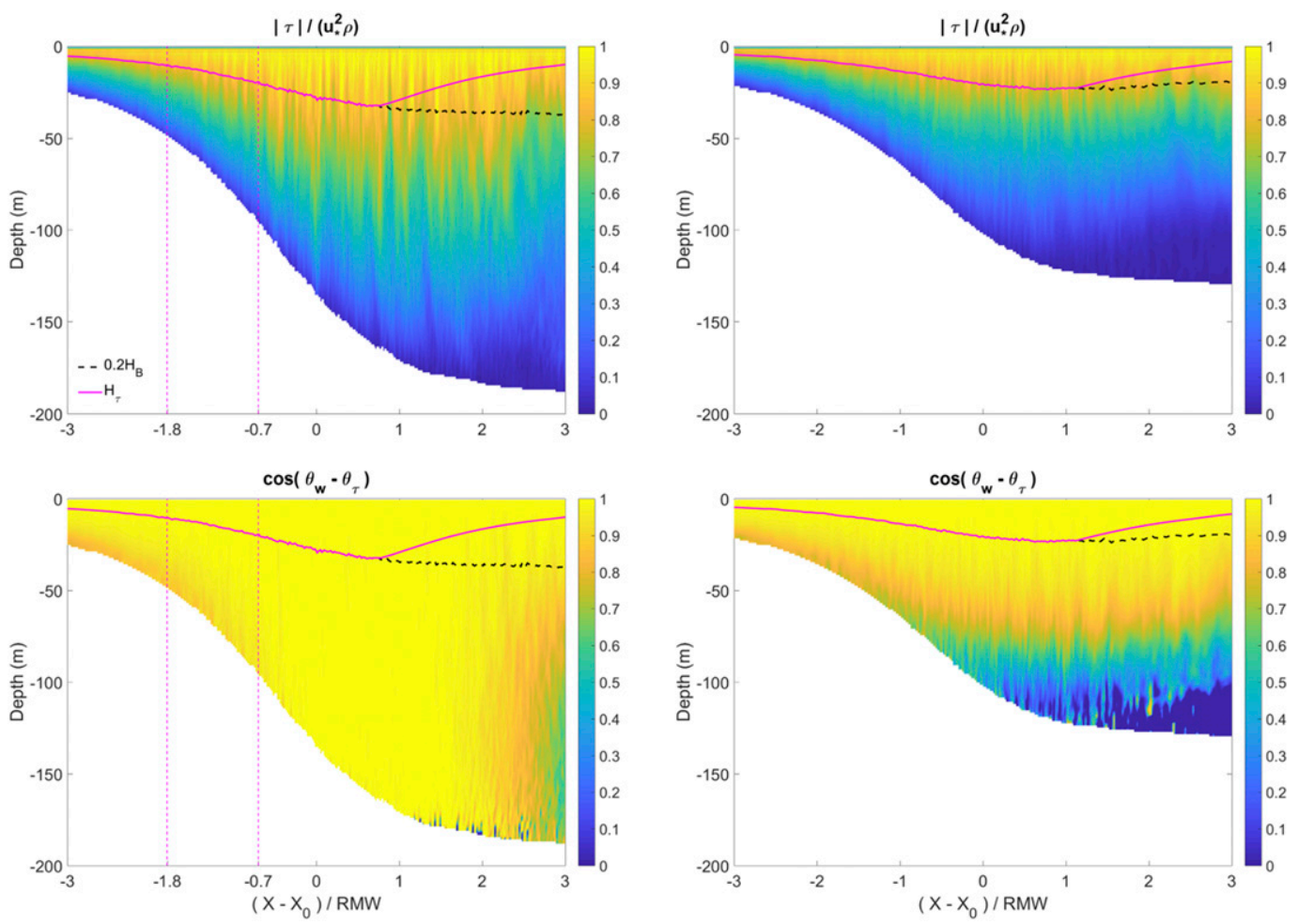

FIG. 3. (top) Normalized Reynolds stress $|\tau| /\left(u_{*}^{2} \rho\right)$ and (bottom) the misalignment between Reynolds stress's direction and the wind direction for the transect on (left) $\left(Y-Y_{0}\right) / \mathrm{RMW}=1$ and (right) $\left(Y-Y_{0}\right) / \mathrm{RMW}=-1.2$. The solid magenta line denotes the depth of the layer with substantial stress $\left(H_{\tau}\right)$ and the dashed black line is the upper $20 \%$ of boundary layer depth $\left(0.2 H_{B}\right)$. Note that $H_{\tau}=0.2 H_{B}$ only in the strongly wind-forced subregion (refer to Fig. 4), which corresponds to the range $\left(X-X_{0}\right) / \mathrm{RMW}<1$ in the left panels and $\left(X-X_{0}\right) / \mathrm{RMW}<-1$ in the right panels. The vertical dashed red line denotes the range of mean current shear and stress profiles that will be examined in Fig. 5 .

since $|\boldsymbol{\tau}|$ normalized by $u_{*}^{2} \rho$ at $z=-0.2 H_{B}$ is close to one. These results suggest that there exists a near-surface layer where $\boldsymbol{\tau}$ is nearly constant in the vertical.

Figure 3 shows the evolution of Reynolds stress profiles for two transects: one is on the right of the TC track at $\left(Y-Y_{0}\right) / \mathrm{RMW}=1$ and the other one is on the left at $\left(Y-Y_{0}\right) / \mathrm{RMW}=-1.2$ (black dashed lines in Fig. 2). For both transects, $\boldsymbol{\tau}$ does not decay significantly within upper $0.2 H_{B}$ and remains nearly constant $\left[|\boldsymbol{\tau}| /\left(u_{*}^{2} \rho\right)>0.8\right]$ (top panels, Fig. 3). However, $\tau$ remains aligned with the wind direction down to a much greater depth than the layer with substantial stress. This alignment is especially true for the right-side transect, where the wind-aligned $\tau$ approaches the boundary layer base (bottom panels, Fig. 3). This indicates that $\boldsymbol{\tau}$ does not simply follow the Ekman spiral structure for which $\tau$ decays and rotates at the same rates with depth. Thus, it is clear that the spatiotemporal variation of TC's wind forcing may also significantly impact the stress profile.

To facilitate understanding of the stress profile and quantitatively specify the depth of the layer with substantial stress $H_{\tau}$, we assess $\tau$ through the horizontally averaged momentum equation after subtracting geostrophic currents without using parameterized turbulent eddy viscosity:

$$
\frac{\partial\langle\mathbf{u}\rangle}{\partial t}+f \hat{\mathbf{z}} \times\left(\langle\mathbf{u}\rangle+\mathbf{u}_{s}\right)=\frac{1}{\rho} \frac{\partial \tau}{\partial z},
$$

where $\mathbf{u}=(u, v)$ is resolved horizontal Eulerian current vector with components along east and north directions, $\langle\cdot\rangle$ denotes the horizontal spatial average in the LES domain, $\hat{\mathbf{z}}$ is a unit vector in the $z$ direction, and $f=$ $6.81 \times 10^{-5} \mathrm{~s}^{-1}$ is the Coriolis parameter. To indicate the variation of the dominant forcing in Eq. (2), we compute the ratio $R_{M}$ of the depth-integrated current acceleration rate [first term on the lhs of Eq. (2)] to the depth-integrated Coriolis force [second term on the lhs of Eq. (2)] following

$$
R_{M}=\frac{\left|\int_{-H}^{0} \partial\langle\mathbf{u}\rangle / \partial t d z\right|}{\left|\int_{-H}^{0} f \hat{\mathbf{z}} \times\left(\langle\mathbf{u}\rangle+\mathbf{u}_{s}\right) d z\right|},
$$



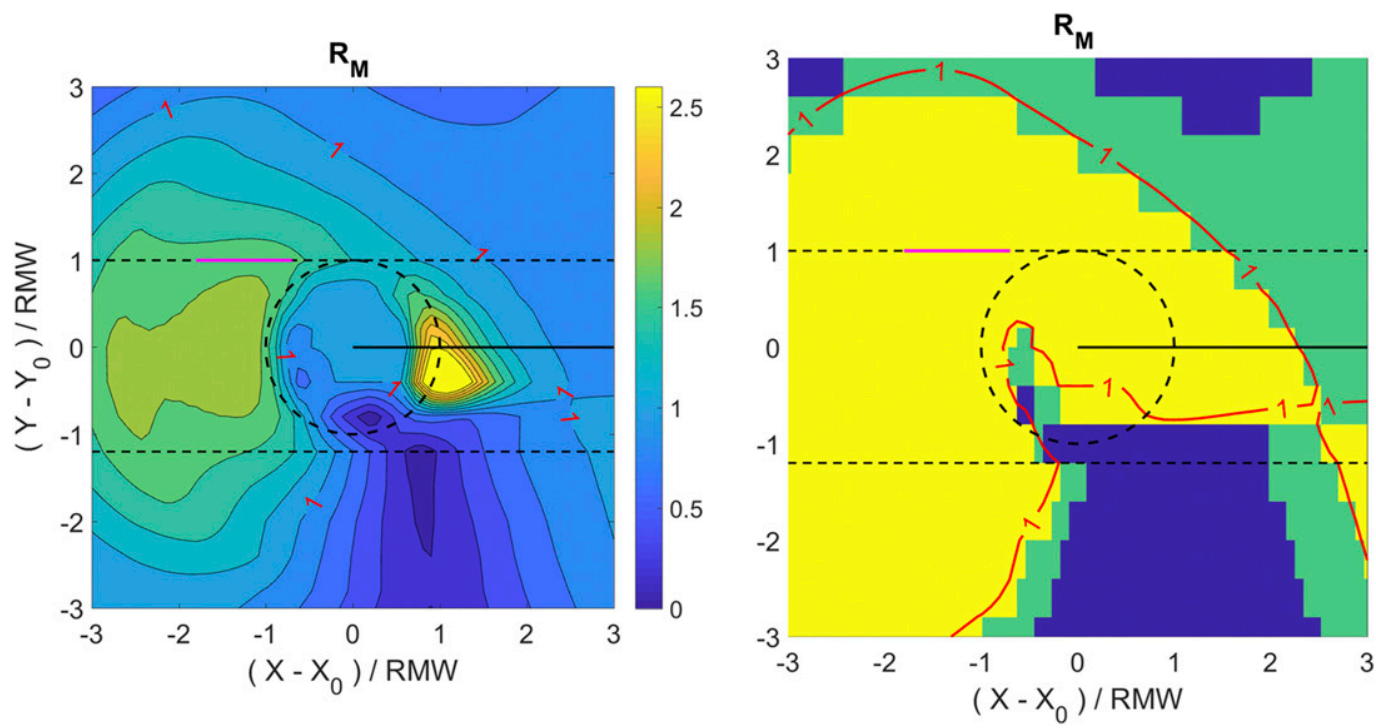

FIG. 4. (left) The ratio $R_{M}$ of depth-integrated mean current acceleration rate to the depth-integrated Coriolis force. (right) The strongly wind-forced (yellow region), inertial oscillation (green region), and Ekman layer (blue region) regimes based on $R_{M}$. The red contour line in the right panel denotes $R_{M}=1$. Other line styles are the same as in Fig. 2.

where $H$ is the depth of the LES domain (Fig. 4). Physically, $R_{M}$ characterizes the ratio of the inertial period to the time scale of the mean current change. When $R_{M}$ is about 1 , the momentum balance is mainly between the acceleration of the mean currents and the Coriolis force, indicating inertial oscillation. We identify strong inertial currents for $0.8<R_{M}<1$, which corresponds to regions on the right-side after the TC's passage (shown as green regions in the right panel, Fig. 4).

We expect that the profile of $\tau$ varies with different dominant forcing. However, $\tau$ is always close to the wind stress sufficiently close to the surface. We define the depth of the layer with substantial stress as $H_{\tau}$ for all three forcing regimes. Note that we mainly introduce a constant stress layer for scaling purposes, so that the stress is only approximately constant with depth in this layer. The proper scaling of $H_{\tau}$ depends on the particular forcing subregion, which is investigated next.

When $R_{M}>1$ the primary momentum balance is between the acceleration of the mean currents and the divergence of $\tau$ (regions with $R_{M}>1$ in the left panel, also shown as yellow regions in the right panel, Fig. 4). In this case, the mean currents change faster than the inertial oscillation and thus are also substantially forced by the wind. This scenario yields a profile of $\boldsymbol{\tau}$ that is mostly aligned with wind throughout $H_{B}$ (as shown for $\left(X-X_{0}\right) /$ RMW $<1$ in the bottom-left panel and for $\left(X-X_{0}\right) /$ RMW $<-1$ in the bottom-right panel, Fig. 3). To estimate a depth to which $\tau$ is approximately constant $\left(H_{\tau}\right)$, we assume a steady mean current shear within $H_{B}$, and as such

$$
0 \approx \frac{\partial}{\partial t}\left(\frac{\partial\langle\mathbf{u}\rangle}{\partial z}\right)=\frac{1}{\rho} \frac{\partial^{2} \boldsymbol{\tau}}{\partial z^{2}},
$$

yielding a linearly decaying profile of $\tau$.

To assess this assumption, we examine the profiles of mean Eulerian current shear and $\boldsymbol{\tau}$ between $\left(X-X_{0}\right) / \mathrm{RMW}=-1.8$ and $\left(X-X_{0}\right) / \mathrm{RMW}=-0.7$ at station $\left(Y-Y_{0}\right) / \mathrm{RMW}=1$, which are located in the strongly wind-forced region for both the LT and ST cases. To illustrate the direction of the mean Eulerian current and its shear relative to the wind, both the current and shear are projected into the along-wind and crosswind directions denoted by subscripts // and $\perp$, respectively.

For both the LT and ST cases, the along-wind part of the mean Eulerian current (black solid and black dashed lines, Figs. 5a,e) and shear (Figs. 5b,f) are predominant compared to the crosswind part (red solid and red dashed lines, Figs. 5a,e,c,g) since the Coriolis effect is not significant in the strongly wind-forced region. In spite of the rapidly growing TC winds and significantly increased along-wind mean Eulerian currents (cf. black dashed to black solid lines, Figs. 5a,e), the shear profiles rarely change (Figs. 5,b,c,f,g), which supports our steady mean shear assumption. Note that shear in Fig. 5 is plotted as function of $z / H_{B}$ and that $H_{B}$ changes with time; however, the boundary layer deepens relatively slowly, about $0.003 \mathrm{~m} \mathrm{~s}^{-1}$, and is relatively deep with an average depth of about $80 \mathrm{~m}$, so that changes in $H_{B}$ do not significantly affect the temporal shear evolution shown in Fig. 5. As expected, $\tau$ decreases linearly for both the LT and ST cases (Figs. 5d,h). For this regime, 

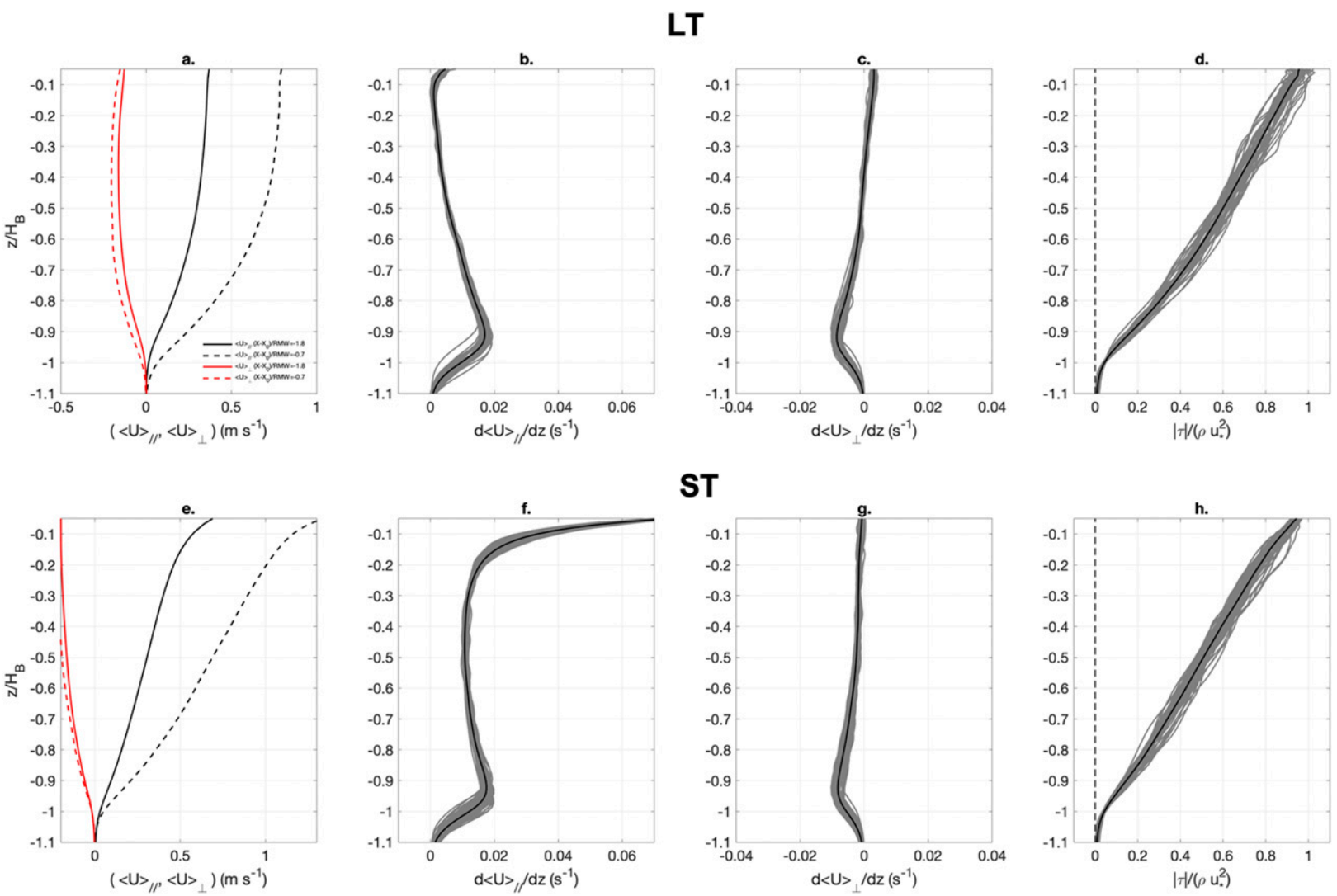

FIG. 5. (a),(e) The along-wind (black lines) and crosswind (red lines) mean Eulerian currents at $\left(X-X_{0}\right) / \mathrm{RMW}=-1.8$ (solid lines) and at $\left(X-X_{0}\right) / \mathrm{RMW}=-0.7$ (dashed lines) for the (top) LT and (bottom) ST cases. (b),(f) The along-wind mean Eulerian current shear profiles and their mean, (c),(g) the crosswind mean Eulerian current shear profiles and their mean, (d),(h) the Reynolds stress profiles and their mean between $\left(X-X_{0}\right) / \mathrm{RMW}=-1.8$ and $\left(X-X_{0}\right) / \mathrm{RMW}=-0.7$ for the (top) LT and (bottom) ST cases. Gray lines denote each single profile, and the black line is the mean in (b), (c), (d), (f), (g), and (h).

we set $H_{\tau}=0.2 H_{B}$, so that $\tau$ decays to $80 \%$ of surface value at $z=H_{\tau}$ (solid magenta lines in the strongly windforced subregion, which is for $\left(X-X_{0}\right) / \mathrm{RMW}<1$ in the left panels and for $\left(X-X_{0}\right) / \mathrm{RMW}<-1$ in the right panels, Fig. 3).

For $R_{M}<0.8$, the primary momentum balance is between the Coriolis force and the divergence of $\tau$, which forms the classic Ekman layer with a spiral structure of current and $\tau$ (regions with $R_{M}<0.8$ in the left panel, also shown as blue regions on the left side of the TC in the right panel, Fig. 4). We consider $\tau$ changes little vertically within $H_{\tau}$ and is scaled by $u_{*}^{2} \rho$, which reveals

$$
f\left(|\mathbf{u}\rangle+\mathbf{u}_{s} \mid\right) \sim \frac{u_{*}^{2}}{H_{\tau}} .
$$

Since depth-averaged $\left|\mathbf{u}_{s}\right|$ over $H$ is smaller than the depth-averaged $|\langle\mathbf{u}\rangle|$ on the left side of the TC (refer to Fig. 7 in Wang et al. 2018) and $\langle\mathbf{u}\rangle$ is still significantly forced by the winds, the depth-averaged Lagrangian currents $\left(\langle\mathbf{u}\rangle+\mathbf{u}_{s}\right)$ can be approximately scaled by $u *$, resulting in $H_{\tau} \sim u_{*} / f$. We find that $H_{\tau}=0.025 u * / f$ best captures the depth of the layer with substantial stress (solid magenta lines for $\left(X-X_{0}\right) /$ RMW $>1$, Fig. 3). Note that here $H_{\tau}=0.025 u * / f$ is obtained through scaling the momentum equation and is not the Ekman depth scale, which is also suggested by the relatively small coefficient 0.025 compared to the typical coefficient (0.25) in the Ekman depth scale (McWilliams et al. 1997). In addition, $\tau$ is not exactly constant within $H_{\tau}$ so that the divergence of $\boldsymbol{\tau}$ is not zero and can still balance the relatively weak Coriolis force due to the less energetic inertial currents on the left side of the TC.

In summary, the characteristics of the stress profile vary with the dominant forcing under the TC and thereby leading to different scalings for $H_{\tau}$. For a conservative estimate in our study, $H_{\tau}=\min \left(0.2 H_{B}, 0.025 u_{*} / f\right)$, which should also include the inertial oscillation case because the inertial oscillation does not influence $\tau$ 's profile (solid magenta lines, Fig. 3). In the next section, we first investigate the Lagrangian shear, which critically determines LT direction. We then examine the Stokes 

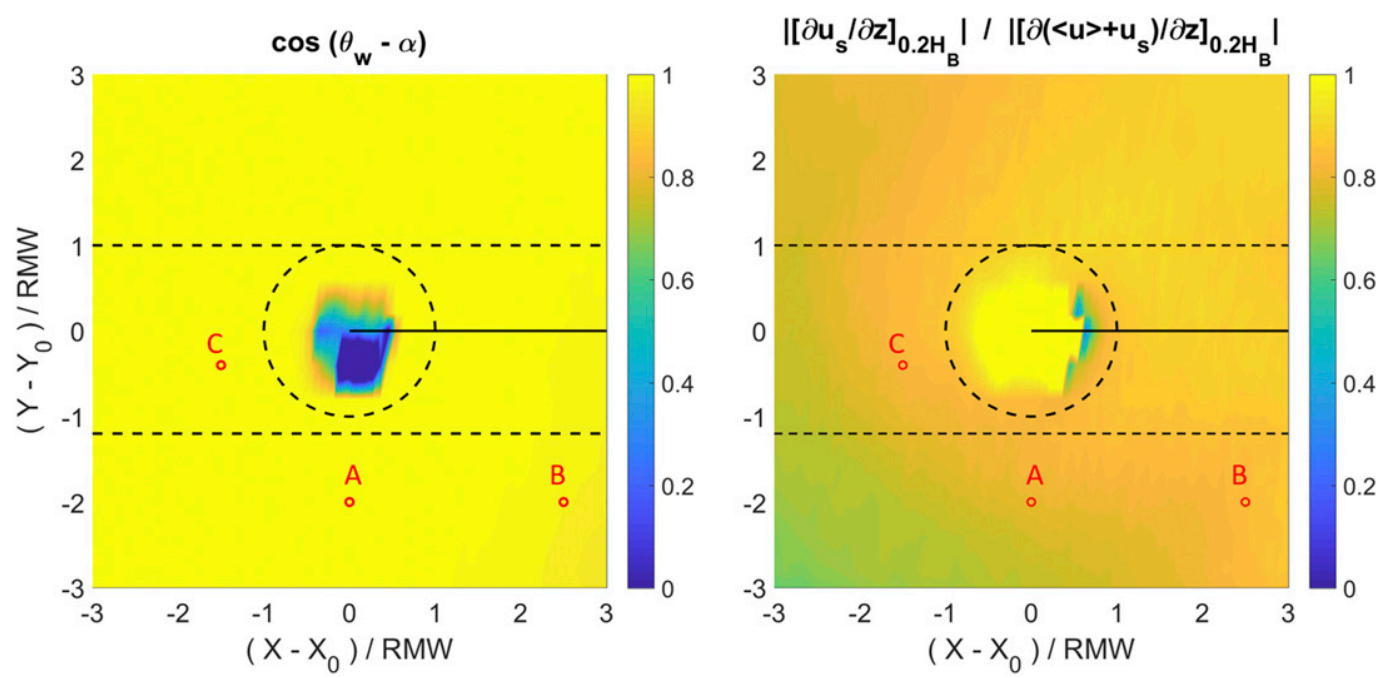

FIG. 6. (left) The cosine of misalignment angle between the directions of wind and surface layer-averaged Lagrangian shear. (right) The ratio of the magnitude of surface layer-averaged Stokes drift shear to the magnitude of surface layer-averaged Lagrangian shear. The surface layer average is conducted within the upper $0.2 H_{B}$. Letters A, B, and C denote three locations as in Fig. 1, and other line styles are the same as in Fig. 2.

drift shear, which predominantly contributes to the Lagrangian shear and controls Stokes drift shear production with the Reynolds stress.

\section{b. Wind-aligned Lagrangian shear and Stokes drift shear}

Earlier studies show that LT direction is determined by a depth-averaged Lagrangian shear (DALS):

$$
\text { DALS }=\left[\frac{\partial\langle\mathbf{u}\rangle}{\partial z}\right]_{0.2 H_{B}}+\left[\frac{\partial \mathbf{u}_{S}}{\partial z}\right]_{0.2 H_{B}},
$$

where the symbol $[\cdot]_{0.2 H_{B}}$ denotes the depth average within the upper $0.2 H_{B}$. The DALS direction $\alpha$ is defined by

$$
\tan (\alpha)=\frac{\left[\partial\left(\langle\boldsymbol{v}\rangle+v_{s}\right) / \partial z\right]_{0.2 H_{B}}}{\left[\partial\left(\langle u\rangle+u_{s}\right) / \partial z\right]_{0.2 H_{B}}},
$$

where $\left(u_{s}, v_{s}\right)=\mathbf{u}_{s}$ are components of the Stokes drift vector along east and north directions (Van Roekel et al. 2012; Sullivan et al. 2012). In spite of TC's substantial wind-wave misalignments (refer to Fig. 1), DALS exhibits a good alignment with the wind in most of the TC domain except for regions near the TC eye, which suggests wind-aligned LT under the TC (left panel, Fig. 6). To further understand the wind-aligned DALS, we examine $[\partial\langle\mathbf{u}\rangle / \partial z]_{0.2 H_{B}}$ and $\left[\partial \mathbf{u}_{s} / \partial z\right]_{0.2 H_{B}}$, which are two components of the DALS [Eq. (6)]. The ratio of $\left|\left[\partial \mathbf{u}_{s} / \partial z\right]_{0.2 H_{B}}\right|$ to $|\mathrm{DALS}|$ is greater than 0.8 in most of the TC domain except in regions on the lhs before the TC eye passes (right panel, Fig. 6), where fast propagating, wind-misaligned long waves are predominant with relatively small Stokes drift shear [refer to the bottom left panel of Fig. 1 in Wang et al. (2018)]. This indicates that $\left[\partial \mathbf{u}_{s} / \partial z\right]_{0.2 H_{B}}$ determines DALS in most regions under TC. Thus, the wind-aligned DALS is controlled by the wind-aligned $\left[\partial \mathbf{u}_{s} / \partial z\right]_{0.2 H_{B}}$. Note that $\left[\partial \mathbf{u}_{s} / \partial z\right]_{0.2 H_{B}}$ emphasizes surface Stokes drift contributions; however, $\partial \mathbf{u}_{s} / \partial z$ is potentially depth dependent, which will be examined below.

To illustrate the directional profile of $\partial \mathbf{u}_{s} / \partial z$, we compare it to the wind direction at the same two transects in section 3a (denoted by two dashed black lines in Fig. 2). For both transects, $\partial \mathbf{u}_{s} / \partial z$ is found to be well aligned with the wind at $z>-H_{\tau}$ before the TC's passage [ $\left(X-X_{0}\right) /$ RMW $<0$; top panels, Fig. 7]. During maximum winds, the wind-aligned Stokes drift shear even extends to a depth that is greater than $50 \mathrm{~m}$ due to the strongly wind-forced, mature waves with longer wavelengths $\left(0<\left(X-X_{0}\right) / \mathrm{RMW}<1\right.$, top left panel, Fig. 7). The pronounced misalignment mostly occurs before and after the TC's passage at $z<-H_{\tau}$. This is because most wind-misaligned swell waves occur during those stages and become more pronounced in a deep layer $\left[\left(X-X_{0}\right) /\right.$ RMW $<-1$ and $\left(X-X_{0}\right) /$ RMW $>2$; top panels, Fig. 7].

Considering the layer with substantial stress $\left(H_{\tau}\right)$ described in section 3a, our results show that the Reynolds stress and the Lagrangian shear, which approximately equals $\partial \mathbf{u}_{s} / \partial z$, are both along the wind direction within depth $H_{\tau}$. However, both are aligned with the wind for different reasons: the Lagrangian shear is controlled by 

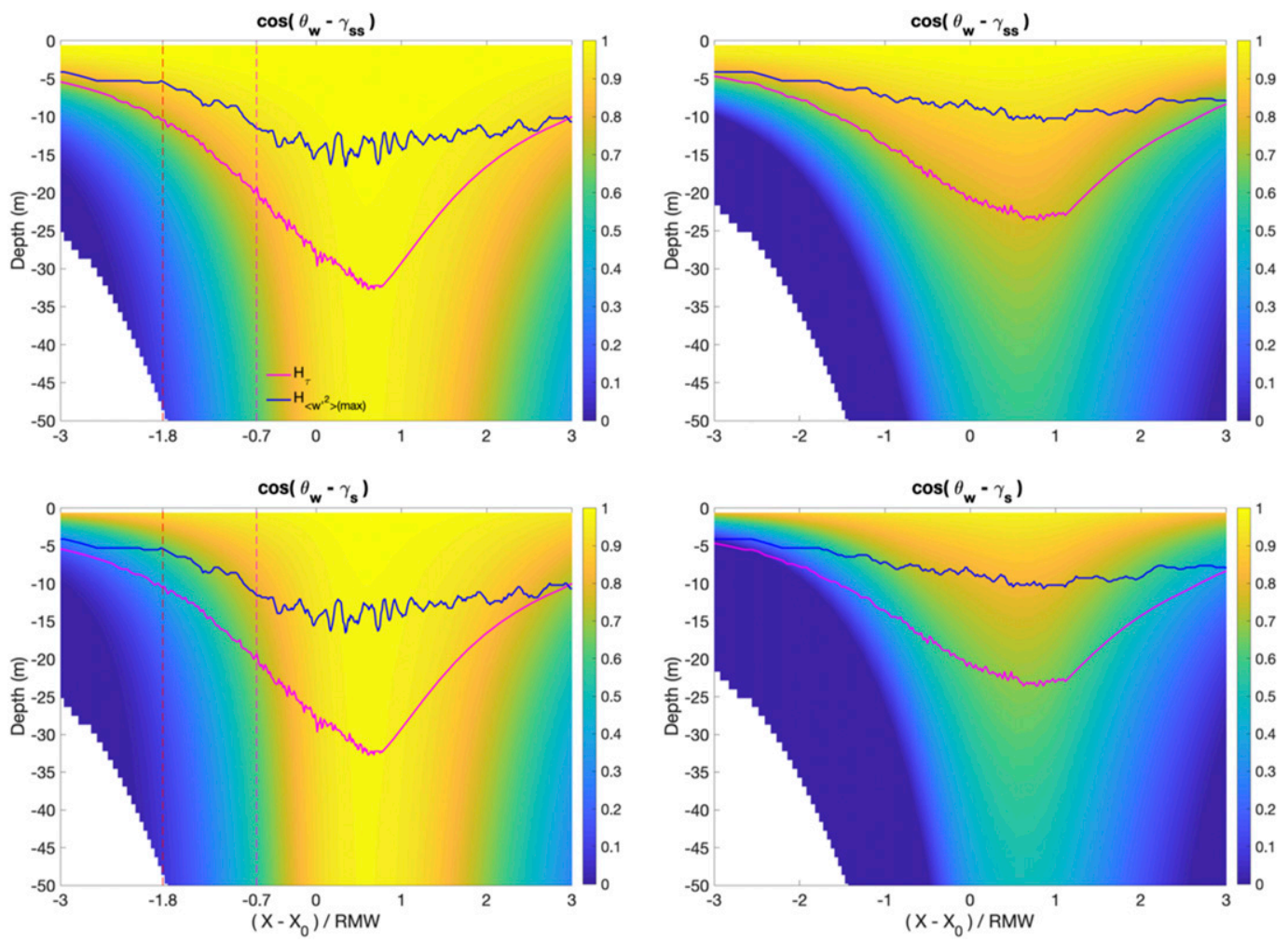

FIG. 7. (top) The cosine of the misalignment between the Stokes drift shear direction $\gamma_{\mathrm{ss}}$ and the wind direction $\theta_{w}$ and (bottom) the misalignment between the Stokes drift direction $\gamma_{s}$ and the wind direction $\theta_{w}$ on the (left) rhs transect and (right) lhs transect. The solid blue line indicates the depth of maximum $\left\langle w^{2}\right\rangle H_{\left\langle w^{\prime 2}\right\rangle(\max )}$, and the solid magenta line denotes the depth of $H_{\tau}$. The vertical dashed red line denotes the range of mean current shear and stress profiles examined in Fig. 5.

wind-forced waves, while the Reynolds stress is dictated by the momentum budget, irrespective of the Lagrangian shear direction. This finding is particularly interesting in light of recent parameterizations of the Reynolds stress (e.g., McWilliams et al. 2014; Reichl et al. 2016a), whose direction is assumed along the Lagrangian shear in the presence of LT. Is this directional dependence causal or is a more comprehensive approach necessary to parameterize the Reynolds stress, for example, one that includes nonlocal transport?

One characteristic of nonlocal momentum transport is the presence of relatively weak Lagrangian mean shear and substantial vertical momentum flux, so that momentum is not simply transported downgradient along the Lagrangian mean shear. As an example, we examine the magnitude of the normalized along-wind Lagrangian shear in the presence of LT at $\left(Y-Y_{0}\right) / \mathrm{RMW}=1$ and compare it to the ST case (Fig. 8). The crosswind Lagrangian shear is much weaker than the along-wind part for both cases and is not shown here. As expected, the magnitude of the Lagrangian shear is most pronounced near the surface and bottom due to the predominant surface Stokes drift shear and bottom Eulerian current shear, respectively (Fig. 8). The bottom Eulerian current is relatively weak before the TC passes $\left[\left(X-X_{0}\right) /\right.$ RMW $\left.<-1\right]$, resulting in a small bottom Lagrangian shear at that stage. The magnitude of the normalized Lagrangian shear for the LT case is quite weak in a middle layer $\left(-0.4 H_{B}<z<-H_{\tau}\right)$ compared to the ST case, where $\tau$ is still significant. If the turbulent flux is parameterized based on the downgradient assumption, such relatively weak shear results in a relatively large, that is, enhanced, eddy viscosity (McWilliams and Sullivan 2000; Reichl et al. 2016b). Previous studies suggest that such an enhancement may also capture nonlocal transport (Smyth et al. 2002; Kukulka et al. 2012; Sinha et al. 2015). Additional evidence for nonlocal transport is presented in section $3 \mathrm{e}$, indicating that dominant Langmuir cells contribute to a significant portion of momentum transport.

For a comparison to the directional profiles of $\partial \mathbf{u}_{s} / \partial z$, we also assess the directional profile of $\mathbf{u}_{s}$, which shows considerable misalignment with the wind within $H_{\tau}$, particularly before and after the TC's passage (bottom panels, Fig. 7). This is because $\partial \mathbf{u}_{s} / \partial z$ is weighted more by the wind-aligned short waves with greater wavenumber 

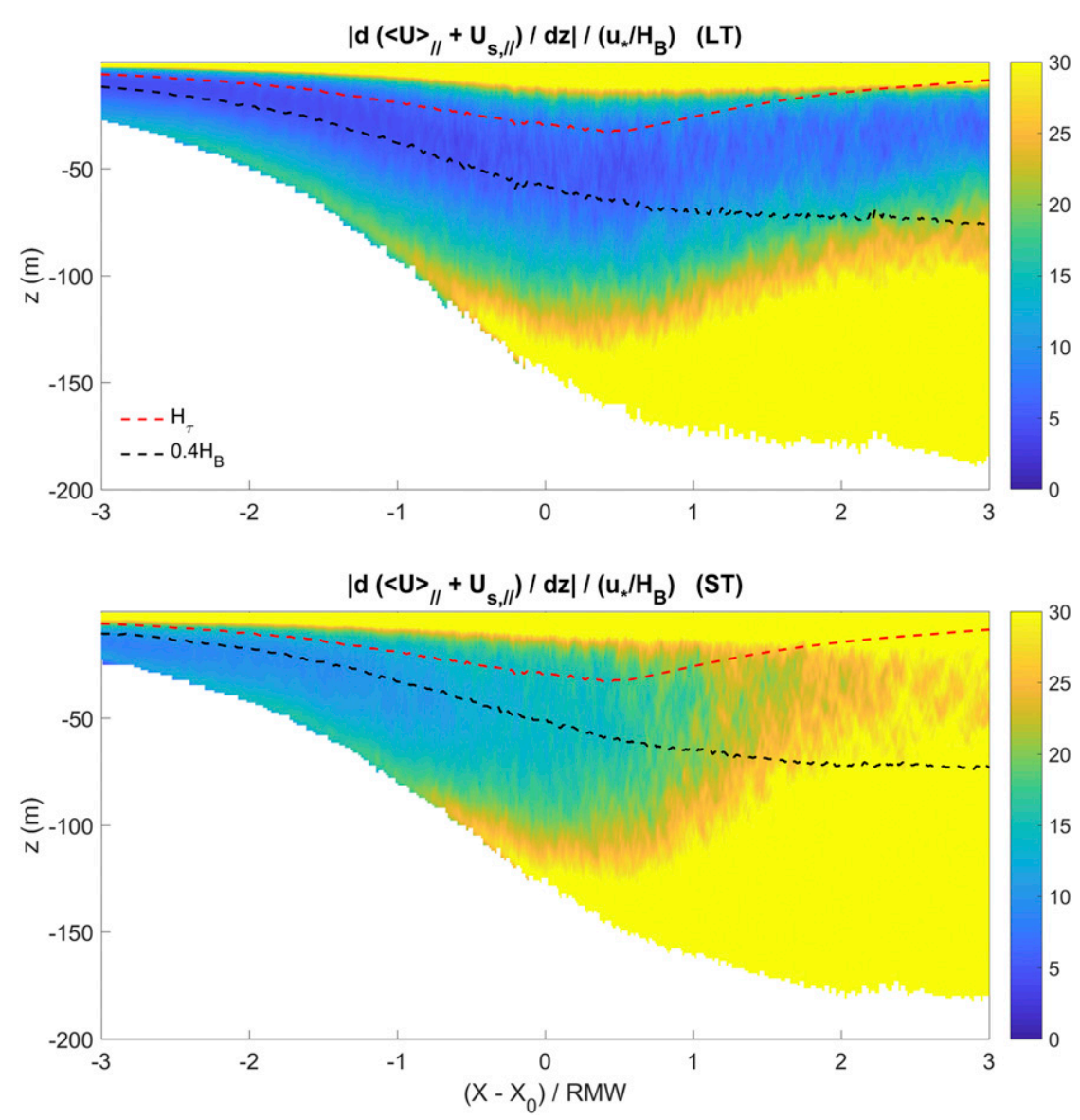

FIG. 8. Transects of the normalized along-wind Lagrangian shear magnitude at $\left(Y-Y_{0}\right) / \mathrm{RMW}=1$ for the (top) LT case and (bottom) ST case. The Lagrangian shear is normalized by $u_{*} / H_{B}$, where $u_{*}$ is water friction velocity and $H_{B}$ is the boundary layer depth. The red dashed line indicates $H_{\tau}$, and the black dashed line denotes the depth of $0.4 H_{B}$.

that tend to be more aligned with the wind than $\mathbf{u}_{s}$ [refer to Eq. (1)]. Though the wind-misaligned long waves barely affect the direction of wind-aligned $\partial \mathbf{u}_{s} / \partial z$, it can generate pronounced, wind-misaligned $\mathbf{u}_{s}$ at greater depth because of its relatively slow decay rate. Thus, we emphasize that the directional variability of $\mathbf{u}_{s}$ is different from $\partial \mathbf{u}_{s} / \partial z$. It is the misalignment between the wind and $\mathbf{u}_{s}$ within $H_{\tau}$ that accounts for the reduced LT intensity, which will be shown in section $3 \mathrm{~d}$.

We next filter the wind-forced wave components from the original wave spectrum to examine whether relatively short waves that are directly forced by the wind contribute to the alignment of Stokes drift shear with wind. We employ a wave-age criteria for the filter:

$$
0<\frac{c}{\left|\mathbf{U}_{10}\right| \cos (\Delta \theta)}<1.2
$$

where $c$ is the phase speed and $\Delta \theta$ is $\Delta \theta=\theta-\theta_{w}$ (Portilla et al. 2009; Kukulka et al. 2017). This criterion is applied to each wave component in the directional wave spectrum $F(\omega, \theta)$. We can thus compute the corresponding depth-averaged Stokes drift shear $\left[\partial \mathbf{u}_{s}^{w} / \partial z\right]_{0.2 H_{B}}$ for the wind-forced wave spectra and compare it to $\left[\partial \mathbf{u}_{s} / \partial z\right]_{0.2 H_{B}}$ to estimate how much the wind-forced waves contribute to the Stokes drift shear profile in the upper $0.2 H_{B}$. We find that the ratio of $\left[\partial \mathbf{u}_{s}^{w} / \partial z\right]_{0.2 H_{B}}$ to $\left[\partial \mathbf{u}_{s} / \partial z\right]_{0.2 H_{B}}$ is close to one in most regions under the TC except regions near the TC eye, where rapidly turning, decaying winds rarely generate any significant windaligned waves (not shown here). In addition, $\left[\partial \mathbf{u}_{s}^{w} / \partial z\right]_{0.2 H_{B}}$ is as well aligned with the wind throughout most regions of the TC domain. This analysis indicates that the windforced waves predominately contribute to the windaligned Stokes drift shear in the upper $0.2 H_{B}$.

\section{c. Upper layer with predominant Stokes drift shear production}

The preceding sections have systematically investigated the profiles of Reynolds stress $(\boldsymbol{\tau})$ and Stokes drift 

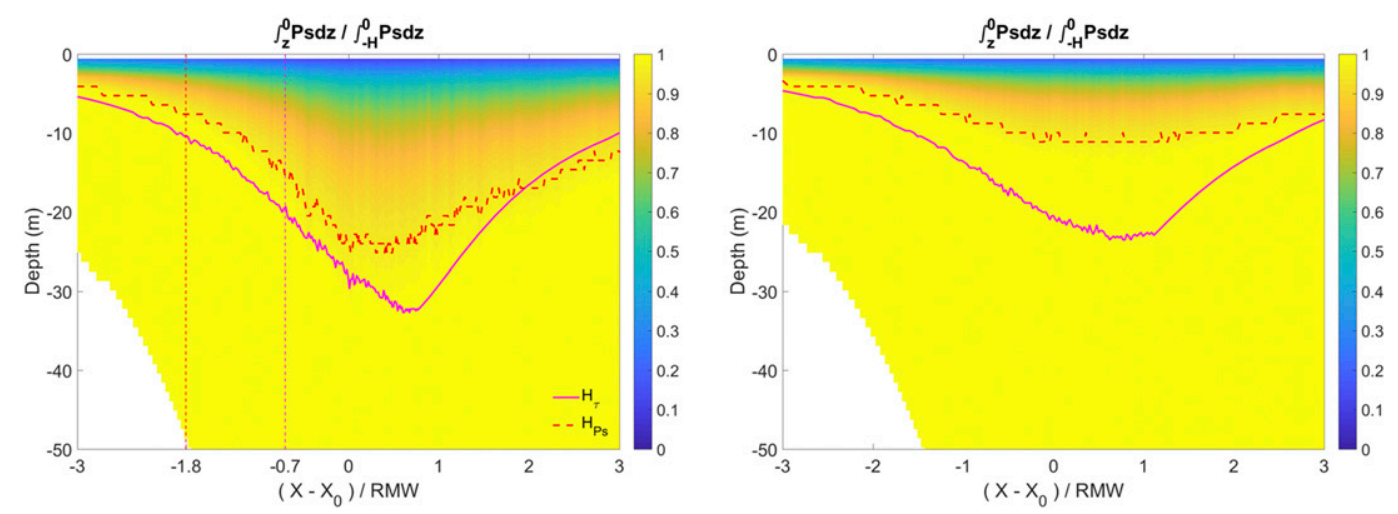

FIG. 9. (top) Depth-accumulated $P_{S}$ that is normalized by the depth-integrated $P_{S}$ throughout the computation depth for the transect on (left) $\left(Y-Y_{0}\right) / \mathrm{RMW}=1$ and (right) $\left(Y-Y_{0}\right) / \mathrm{RMW}=-1.2$. The dashed red line is the depth of predominant Stokes drift shear production layer $H_{P_{s}}$, and the solid magenta line is the depth of the layer with substantial stress $H_{\tau}$. The vertical dashed red line denotes the range of mean current shear and stress profiles examined in Fig. 5.

shear $\left(\partial \mathbf{u}_{s} / \partial z\right)$. These profiles illustrate the layer with substantial stress and the alignment between the Stokes drift shear and the wind in the upper $0.2 H_{B}$. These results facilitate our investigation of the Stokes drift shear production $P_{S}=\tau / \rho \partial \mathbf{u}_{s} / \partial z$, which critically influences LT intensity.

To assess the profile of $P_{S}$, we compute the depthaccumulated $P_{S}, \quad \int_{-z}^{0} P_{S} d z^{\prime}$, and normalize it by $\int_{-H}^{0} P_{S} d z^{\prime}$, where $H$ is the depth of the LES domain. We define a depth $H_{P_{S}}$, at which the normalized $\int_{-z}^{0} P_{S} d z^{\prime}$ equals 0.95 , specifying the upper layer with predominant $P_{S}$ (Fig. 9). We find that $H_{P_{S}}$ varies with the wind-wave forcing and is significantly greater on the right-side transect at $\left(Y-Y_{0}\right) / \mathrm{RMW}=1$ due to stronger waves and LT. For both transects on each side of the TC eye (Fig. 9), $H_{\tau}$ is greater than $H_{P_{S}}$, which suggests that most $P_{S}$ is concentrated within the layer with substantial stress. The ratio of $H_{P_{S}}$ to $H_{\tau}$ is smaller than one throughout most of the TC domain, except for the regions on the right side, at $(Y-$ $\left.Y_{0}\right) / \mathrm{RMW}>2$ with a ratio greater than 1.3 (left panel, Fig. 12). In those regions, the wind-wave forcing is weak and $P_{S}$ below $H_{\tau}$ is negligible.

Therefore, it is reasonable to conclude that the predominant $P_{S}$ mostly concentrates within the layer with substantial stress. As such, the depth-integrated $P_{S}$ is given by

$$
\int_{-H_{B}}^{0} P_{S} d z=\int_{-H_{\tau}}^{0} \frac{\boldsymbol{\tau}}{\rho} \frac{\partial \mathbf{u}_{s}}{\partial z} d z=u_{*}^{2}\left|\mathbf{u}_{s}(0)\right| \cos \left[\theta_{w}-\gamma_{s}(0)\right],
$$

where $\gamma_{s}$ is the direction of $\mathbf{u}_{s}$. Thus, for $\theta_{w} \approx \gamma_{s}(0)$ (surface waves are mostly wind-forced waves, refer to section above), Eq. (9) indicates that the depth-integrated $P_{S}$ is independent from the wind-wave misalignment and is only determined by the magnitude of the surface Stokes drift and the wind stress, although $\mathbf{u}_{s}$ and the wind can be substantially misaligned at greater depth. Note that the direction of $\mathbf{u}_{s}$ is different from its shear direction, and can exhibit considerable amount of misalignments with the wind in the upper $H_{\tau}$ (see, for example, center panel, Fig. 10). This stands in contrast with the intuitive idea that the misalignment between wind stress and Stokes drift at greater depth can reduce the total $P_{S}$, thereby, reducing LT intensity.

To highlight the last point and also to illustrate the effects of the wind-wave misalignment on LT intensity, we investigate LT intensity at two specific locations with significantly different wind-wave misalignments (locations $\mathrm{A}$ and B in Fig. 1). Both locations are at $\left(Y-Y_{0}\right) / \mathrm{RMW}=-2$, but location $\mathrm{A}$ is at $\left(X-X_{0}\right) / \mathrm{RMW}=0$ (during the TC eye's passage $)$, and location $\mathrm{B}$ is at $\left(X-X_{0}\right) / \mathrm{RMW}=2.5$ (after the TC's passage).

LT intensity is often characterized by the depthaveraged VVV:

$$
\mathrm{VVV}=\frac{1}{H_{B}} \int_{-H_{B}}^{0}\left\langle w^{2} d z\right\rangle
$$

(Van Roekel et al. 2012; Rabe et al. 2015; Reichl et al. 2016b). To compare LT intensity at different locations, we use $\left|u_{*}^{2} u_{s}(0) \cos \left[\theta_{w}-\gamma_{s}(0)\right]\right|^{2 / 3}$ to normalize $\mathrm{VVV}$, which relates VVV to the depth-integrated TKE production due to Stokes drift shear (Grant and Belcher 2009). We also checked for both locations $\mathrm{A}$ and $\mathrm{B}$ that TKE production is indeed dominated by Stokes drift shear (e.g., Van Roekel et al. 2012; the projected surface layer number is small in both cases). Such normalized VVV does not depend on total TKE production due to Stokes drift shear, but may still be reduced due to Stokes drift contributions 

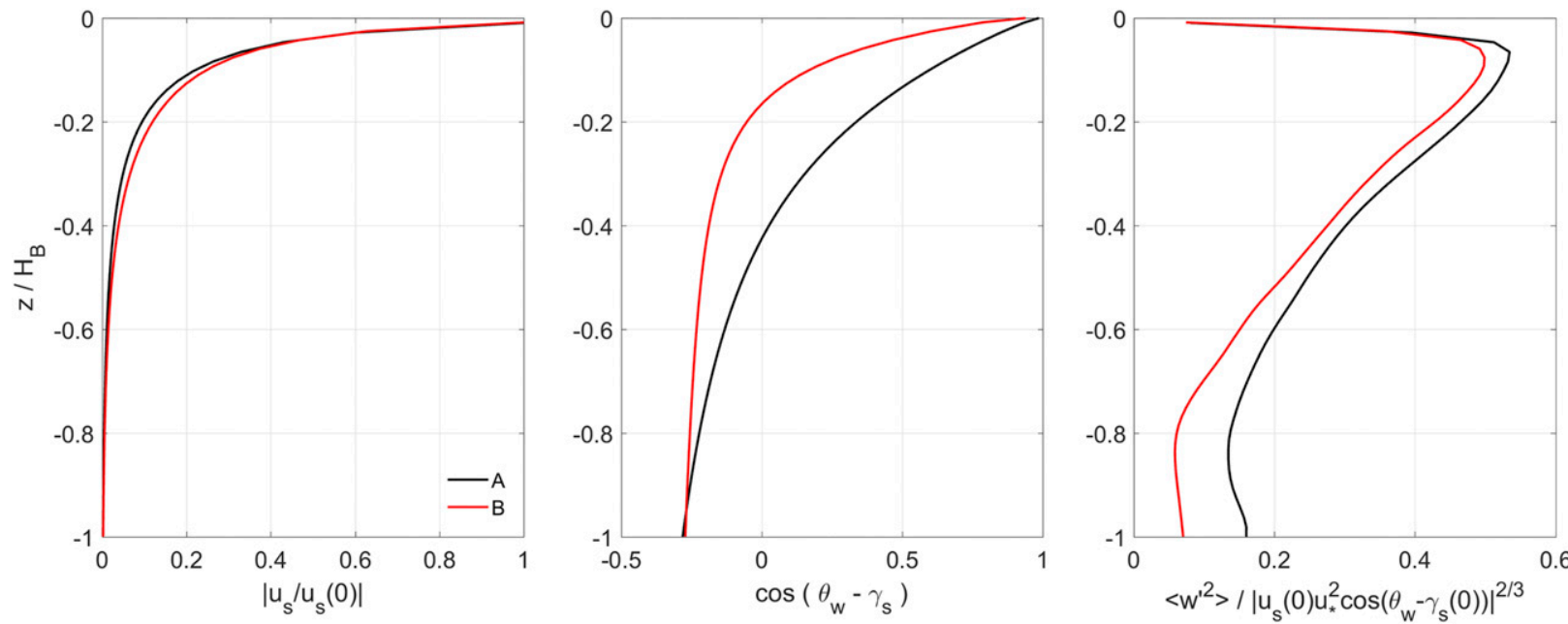

FIG. 10. (left) The normalized vertical profile of $\left|\mathbf{u}_{s}\right|$ by its surface magnitude $\left|\mathbf{u}_{s}(0)\right|$, (center) the cosine of the misalignment between the wind and $\mathbf{u}_{s}$, and (right) the normalized vertical velocity variance by $\left|\mathbf{u}_{s}(0) u_{*}^{2} \cos \left[\theta_{w}-\gamma_{s}(0)\right]\right|^{2 / 3}$ at location A (solid black lines) and location B (solid red lines).

of shorter waves (e.g., Kukulka and Harcourt 2017) or due to deeper misaligned waves as discussed in detail next. Figure 10 (left panel) suggests that faster Stokes drift decay with depth due to greater contribution of shorter waves does not contribute to reduced VVV (right panel in Fig. 10). We find that it is the misaligned Stokes drift at greater depth (middle panel) that mainly causes the VVV reduction apparent in Fig. 10. If the total Stokes drift shear production is the only factor that determines the VVV, we expect that the normalized VVV will be the same for both locations with different wind-wave misalignment. However, we still find a reduced normalized VVV for the case with greater wind-wave misalignment (location $\mathrm{B}$, red line in the center panel, Fig. 10). Therefore, the total Stokes drift shear production alone is not sufficient to explain the reduced VVV, which motivates further investigation of the mechanism by which the wind-wave misalignment reduces LT intensity.

\section{d. Reduced LT intensity due to wind-wave misalignment}

To conceptually illustrate the wind-wave misalignment effect on LT intensity, we employ a simple TKE model based on a balance between Stokes drift shear production $P_{S}=\boldsymbol{\tau} / \rho \partial \mathbf{u}_{s} / \partial z$ and turbulent dissipation $\varepsilon$, which does not necessarily rely on a local balance (Grant and Belcher 2009; Kukulka and Harcourt 2017; Wang et al. 2018). To make further progress, we employ a local assumption to parameterize $\varepsilon$ following previous approaches as $\varepsilon=q^{3} / l$ (Kukulka and Harcourt 2017). Here, $q=$ $(2 \mathrm{TKE})^{1 / 2}$ and $l$ is a mixing length, which is taken to be linearly related to depth here for simplicity. Since we seek to develop a scaling for VVV, which is predominant in the near-surface layer $\left(z>-0.4 H_{B}\right)$ and mostly determined by the Stokes drift shear production, it is reasonable for scaling purposes to only consider the leading order TKE balance between the Stokes drift shear production and dissipation (e.g., Harcourt and D'Asaro 2008; Grant and Belcher 2009; Kukulka and Harcourt 2017).

To associate this simple TKE model to the VVV, we consider a LT-velocity scale $w_{L}$ based on the vertical average of $q^{3}$, which satisfies (assuming TKE production and dissipation are balanced)

$$
w_{L}^{3} \sim \frac{1}{H_{B}} \int_{-H_{B}}^{0} q^{3} d z=\frac{1}{H_{B}} \int_{-H_{B}}^{0} l \frac{\boldsymbol{\tau}}{\rho} \frac{\partial \mathbf{u}_{s}}{\partial z} d z
$$

and $w_{L}^{3} \sim \mathrm{VVV}^{3 / 2}$. In Eq. (11), $l$ reduces $P_{S}$ contributions near the surface, where $l$ and turbulent eddies are smaller, but emphasizes $P_{S}$ contributions at greater depth within $H_{P_{S}}$, where $l$ and turbulent eddies are greater. After integration by parts, Eq. (11) yields

$$
\begin{aligned}
w_{L}^{3} & \sim\left[\frac{l \boldsymbol{\tau} \cdot \mathbf{u}_{s}}{H_{B} \rho}\right]_{-H_{B}}^{0}-\frac{1}{H_{B} \rho} \int_{-H_{B}}^{0} \boldsymbol{\tau} \cdot \mathbf{u}_{s} \frac{\partial l}{\partial z} d z \\
& -\frac{1}{H_{B} \rho} \int_{-H_{B}}^{0} l \mathbf{u}_{s} \frac{\partial \boldsymbol{\tau}}{\partial z} d z .
\end{aligned}
$$

As $l=0$ at the surface and $\mathbf{u}_{s}$ diminishes at the boundary layer base, the first RHS term of Eq. (12) is negligible. Our examinations of the profiles of $\boldsymbol{\tau}$ and $P_{S}$ show that most of $P_{S}$ concentrates in the layer with substantial stress $H_{\tau}$ (refer to sections 3a-c). As such, Eq. (12) can be further simplified as 


$$
w_{L}^{3} \sim-\frac{1}{H_{B}} \int_{-H_{\tau}}^{0} u_{*}^{2}\left|\mathbf{u}_{s}\right| \cos \left(\gamma_{s}-\theta_{w}\right) \frac{\partial l}{\partial z} d z .
$$

The value of $w_{L}^{2} / u_{*}^{2}$ is approximately linearly related to $\mathrm{VVV} / u_{*}^{2}$, which suggests that the simple model Eq. (13) is a useful starting point for understanding VVV scaling relations (Fig. 11). Note that the scaling Eq. (13) is consistent with previous work that empirically relates the normalized VVV to a surface-layer projected Langmuir number (Reichl et al. 2016b), which is approximately proportional to $\left(w_{L}^{2} / u_{*}^{2}\right)^{-3 / 4}$. The outliers in Fig. 11 are mostly from locations in the TC eye region, which are also found in Reichl et al. (2016b) and are possibly associated with the history effects of strongly forced and decaying turbulence under transient forcing (Rabe et al. 2015). Equation (13) also suggests that the wind-projected Stokes drift is the key factor in determining LT intensity and addresses the effects of the wind-wave misalignment on LT intensity.

We further illustrate the effects of the wind-wave misalignment in Eq. (13) through the relation

$$
\int_{-H_{\tau}}^{0}\left|\mathbf{u}_{s}\right| \cos \left(\gamma_{s}-\theta_{w}\right) d z=\cos \left(\theta_{s}-\theta_{w}\right)\left|\int_{-H_{\tau}}^{0} \mathbf{u}_{s} d z\right|,
$$

where $\theta_{s}$ is the direction of $\left[\mathbf{u}_{s}\right]_{H_{\tau}}$ [refer to appendix A in Wang et al. (2018) for the relation between $\gamma_{s}$ and $\theta_{s}$ ]. Thus, the impact of the wind-wave misalignment on LT intensity is addressed by the misalignment between the wind and $\left[\mathbf{u}_{s}\right]_{H_{\tau}}$, which shows a strong spatiotemporal variation (right panel, Fig. 12). The misalignment is small in the regions on the right side of the TC at $\left(X-X_{0}\right) / \mathrm{RMW}>-1$, where LT is also strong [refer to Fig. 9 in Reichl et al. (2016b), indicated by small LasL $\theta$ ]. For regions on the left side at $\left(Y-Y_{0}\right) / \mathrm{RMW}<-1$, the misalignment is great and can be more than $90^{\circ}$ [at $\left(X-X_{0}\right) /$ RMW $<-1$ and $\left(X-X_{0}\right) /$ RMW $>2$ ], suggesting a significant reduction of LT intensity due to wind-wave misalignment. Overall, the misalignment between the wind stress and $\left[\mathbf{u}_{s}\right]_{H_{\tau}}$ varies significantly under the TC, and thereby, plays an important role in affecting LT intensity (e.g., Van Roekel et al. 2012; Rabe et al. 2015; Reichl et al. 2016b). However, in the following section an outstanding question emerges: Langmuir cells are found to be uniformly aligned with the wind direction in spite of TC's great wind-wave misalignment, suggesting that the wind-wave misalignment only reduces LT intensity but does not affect LT direction.

\section{e. Wind-aligned LT}

In this section, we assess the directional variability of LT in TC conditions, particularly, the impact of the

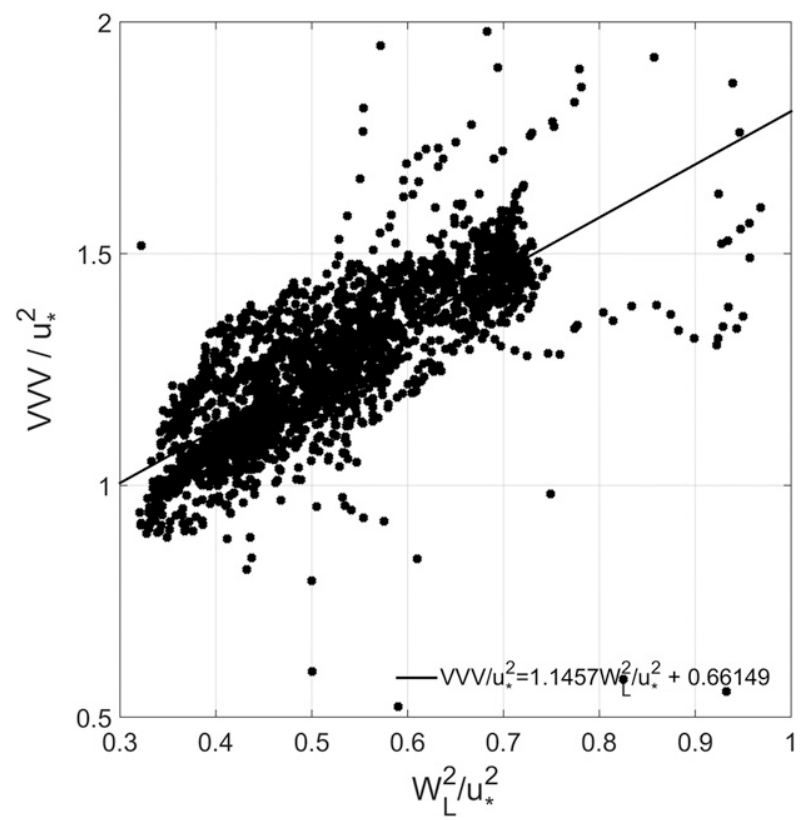

FIG. 11. Normalized vertical velocity variance $\left(\mathrm{VVV} / u_{*}^{2}\right)$ vs normalized $w_{L}^{2} / u_{*}^{2}$ from Eq. (13), with linear regression (solid black line).

wind-wave misalignment on LT direction. First, we introduce an objective method to identify LT direction under transient TC forcing. LT is characterized by coherent vortex pairs that form approximately parallel bands of relatively strong upwelling and downwelling flows (top-left panel, Fig. 13). To identify the direction of such horizontally elongated coherent Langmuir cells in complex wind and wave forcing conditions, we introduce the two-dimensional, horizontal two-point autocorrelation function (Sullivan et al. 2012):

$$
R_{w w}(\Delta x, \Delta y, z, t)=\frac{\langle w(x, y, z, t) w(x+\Delta x, y+\Delta y, z, t)\rangle}{\langle w(x, y, z, t) w(x, y, z, t)\rangle},
$$

where $\Delta x$ and $\Delta y$ are spatial lags in $x$ and $y$ coordinates.

With LT, the autocorrelation function features an elliptic pattern due to elongated turbulent structures (bottom-left panel, Fig. 13) (McWilliams et al. 1997). The orientation of the major axis of the elliptic pattern objectively defines a direction of LT in complex wind and wave conditions. We estimate this direction $\theta_{\mathrm{LT}}$ based on the regression $\Delta y=\tan \left(\theta_{\mathrm{LT}}\right) \Delta x$, where $\Delta x$ and and $\Delta y$ are weighted by $R_{w w}$ (black arrows in Fig. 13). In the ST case, parallel elongated structures are less pronounced and smaller (top-right panel, Fig. 13), which is reflected in $R_{w w}$ with its more circular pattern and greater decay away from the center (bottom-right panel, Fig. 13). The analysis of autocorrelation is performed 

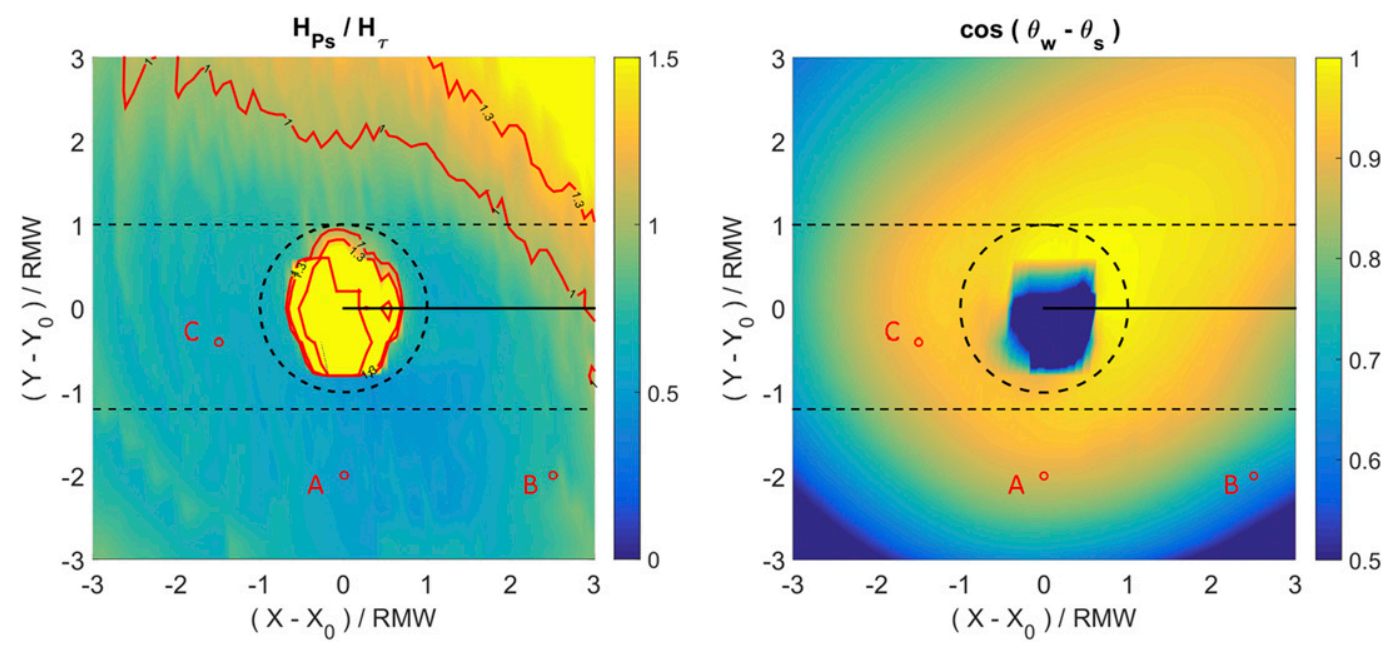

FIG. 12. (left) The ratio of the predominant Stokes drift shear production layer depth $H_{P_{s}}$ to the substantial Reynolds stress layer depth $H_{\tau}$; (right) the misalignment between wind $\theta_{w}$ and depth-averaged Stokes drift over the upper $H_{\tau}\left(\theta_{s}\right)$. The red contours in the right panel denote $H_{P_{s}} / H_{\tau}=1$ and $H_{P_{s}} / H_{\tau}=1.3$. Other line styles are the same as in Fig. 6.

over the full spatial TC extent, which is on a subset of stations and time points for which three-dimensional LES output has been saved (Table 1).

Consistent with earlier studies, LT orientation is aligned with the DALS direction throughout the TC's spatiotemporal domain (Fig. 14). In spite of complex wind and wave conditions under TC, we find that $\theta_{\mathrm{LT}}$ is surprisingly constant over a depth of $0.2 H_{B}$ with a standard deviation of less than $4^{\circ}$ (not shown). This is because the Lagrangian shear, which determines the LT direction, is uniformly aligned with the wind in the upper $0.2 H_{B}$ (refer to section $3 \mathrm{~b}$ ). Therefore, the DALS, which determines LT direction, has the same direction as the Lagrangian shear at each depth above $z=-0.2 H_{B}$.

Given that coherent structures in the form of dominant Langmuir cells (short LCs) are an important feature of LT, it is interesting to examine the Lagrangian shear across the dominant LCs, which plays an important role in setting the LT direction. This is because the along-LC vorticity has its maximum value at the cell center and, thus, the Lagrangian shear at the cell center plays an important role in generating the along-LC vorticity through the vorticity tilting and stretching (Van Roekel et al. 2012).

To find the vortex center of dominant LCs under complex wind-wave conditions, we examine the full three-dimensional structure of dominant LCs with conditional averages following McWilliams et al. (1997) and Kukulka et al. (2010). As strong downwelling jets are common features of LCs (left panel, Fig. 15), we specify a vertical velocity threshold $w<-2\left\langle w^{\prime 2}\right\rangle^{1 / 2}$ at a depth of maximum $\left\langle w^{\prime 2}\right\rangle\left[H_{\left\langle w^{\prime 2}\right\rangle(\max )}\right]$ to identify LC events. For any variable $F(x, y, z, t)$, the conditional average $\{\ldots\}$ is defined as

$$
\{F\}(x, y, z, t)=\frac{1}{n} \sum_{i=1}^{n} F\left(x-x_{i}, y-y_{i}, z, t\right),
$$

where $n$ is the number of LC events and $\left(x_{i}, y_{i}\right)$ specifies the horizontal locations of LC events. For the conditional averaged field $\{F\}$, we define the deviation from the horizontal average as $\{F\}^{\prime}$, which represents the coherent turbulence due to dominant LCs.

We discuss in detail only results for location $\mathrm{C}$ at $\left(X-X_{0}\right) / \mathrm{RMW}=-1.4\left(Y-Y_{0}\right) / \mathrm{RMW}=-0.4($ letter $\mathrm{C}$ in Fig. 1), which are similar to results for other locations (not shown). The map view of $\{w\}$ at $H_{\left\langle w^{\prime 2}\right\rangle(\max )}$ shows strong downwelling jets that coincide with convergent horizontal anomalies $\left(\{u\}^{\prime},\{v\}^{\prime}\right)$ flowing toward the central downwelling region (left panel, Fig. 15). To better illustrate the flow structure, the horizontal velocity anomalies are projected into a plane normal to LT direction, yielding $\{v\}_{\mathrm{LT}}^{\prime}$ (dashed blue line, left panel, Fig. 15), and into an along-LT component, $\{u\}_{\mathrm{LT}}^{\prime}$. In the cross section of dominant LCs, we observe a pair of counterrotating flows for $z>-15 \mathrm{~m}$ (indicated by red arrows, middle panel, Fig. 15). In the center of these two cells there is strong coupling leading to downwelling flow, demonstrating the classic structure of LCs. Our result shows that the center of dominant LCs locates nearly at $H_{\left\langle w^{\prime 2}\right\rangle(\max )}$ and the coherent structures extend to a greater depth than $0.2 H_{B}$, which contributes to the uniform LT direction in the upper $0.2 H_{B}$. Therefore, the Lagrangian shear at $H_{\left\langle w^{\prime 2}\right\rangle(\max )}$ likely influences the direction of dominant LCs. 

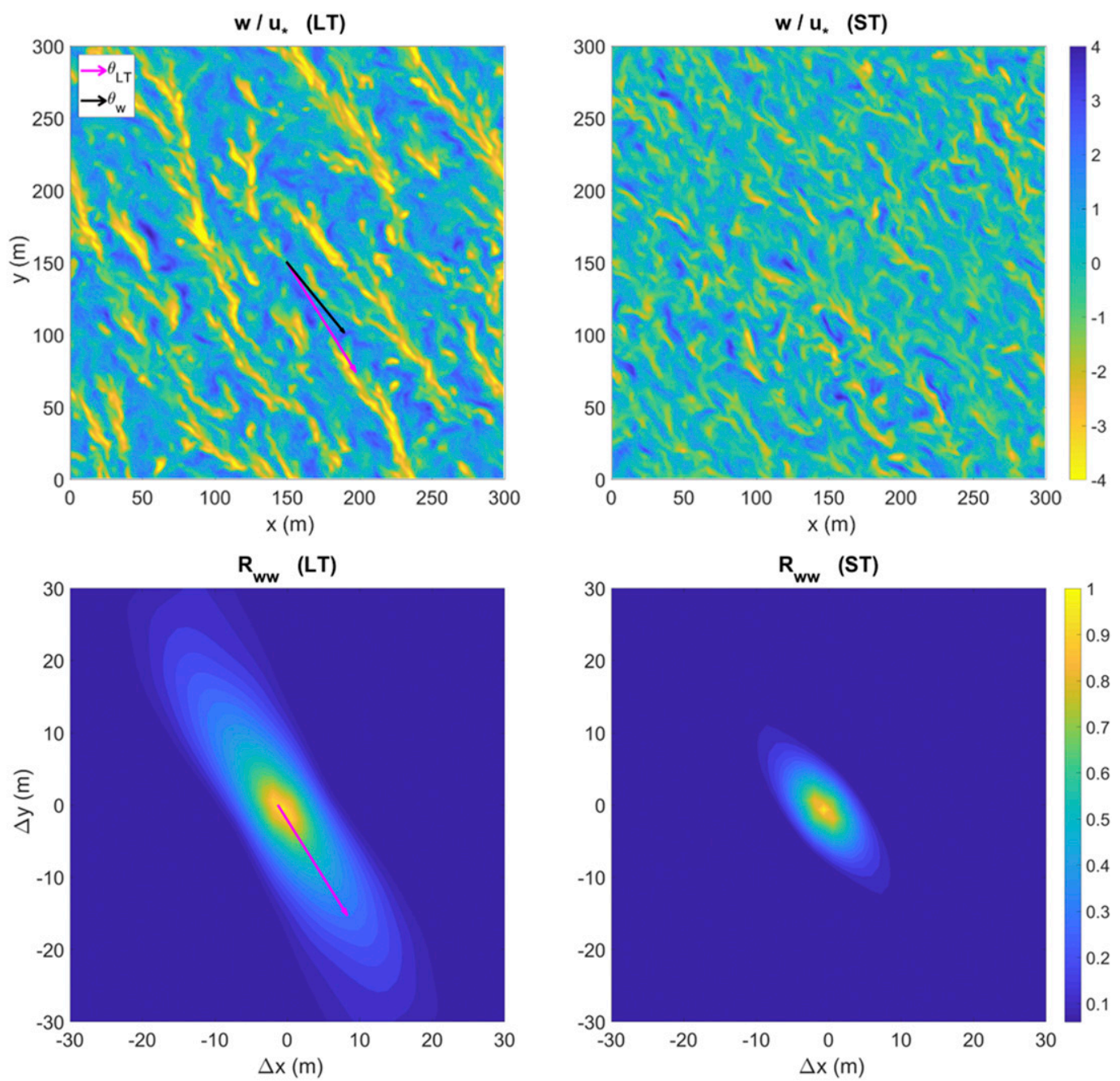

FIG. 13. (top) The horizontal cross section of normalized $w$ at the depth of the maximum vertical velocity variance and (bottom) its corresponding $R_{w w}$ for both the (left) LT case and (right) the ST case at location C, which is at $\left(X-X_{0}\right) / \mathrm{RMW}=-1.4,\left(Y-Y_{0}\right) / \mathrm{RMW}=-0.4$. Note that the horizontal space shows the high-resolution LES space, the magenta arrows in the left panels indicate LT direction, and the black arrow in the top-left panel denotes the wind direction, which is about $7^{\circ}$ misaligned with LT direction.

We find that $H_{\left\langle w^{\prime 2}\right\rangle(\max )}$ is generally shallower than $z=-0.2 H_{B}$, above which the Stokes drift shear is uniformly aligned with the wind (solid blue lines, Fig. 7). This is also true in most of the TC domain except for the region on the right side about two RMW before the TC's passage (Fig. 16). In this region LT rarely occurs because of the great wind-wave misalignment and weak Stokes drift (refer to Fig. 1). Consequently, the Stokes drift shear at $H_{\left\langle w^{\prime 2}\right\rangle(\max )}$ is also aligned with the wind in most of the TC domain. As Stokes drift shear predominantly contributes to the Lagrangian shear, we expect that the Lagrangian shear at $H_{\left\langle w^{\prime 2}\right\rangle(\max )}$ is also aligned with the wind, controlling dominant LCs to be aligned with the wind. Thus, this result shows the consistency between the LT direction and the dominant LCs' direction and contributes explaining why the use of Lagrangian shear at $H_{\left\langle w^{\prime 2}\right\rangle(\max )}$ also well indicates LT direction (Sullivan et al. 2012; Van Roekel et al. 2012).

To provide further evidence for the presence of nonlocal transport in the LT case (see section 3b), we examine the momentum transport due to dominant LCs $\left\langle\{u\}_{\mathrm{LT}}^{\prime}\{w\}\right\rangle$ and $\left\langle\{\boldsymbol{v}\}_{\mathrm{LT}}^{\prime}\{w\}\right\rangle$, which are computed within the span of central downwelling region with a width of a Langmuir cell. We find that dominant LCs substantially transport momentum in the depth ranges between 5 and $20 \mathrm{~m}\left(-0.3 H_{B}<z<-0.05 H_{B}\right)$, where the peak momentum transport contributes to over $40 \%$ of the total momentum transport (right panel, Fig. 15). Previous studies show that this contribution can be more than $50 \%$ (e.g., Kukulka et al. 2010). This further indicates 


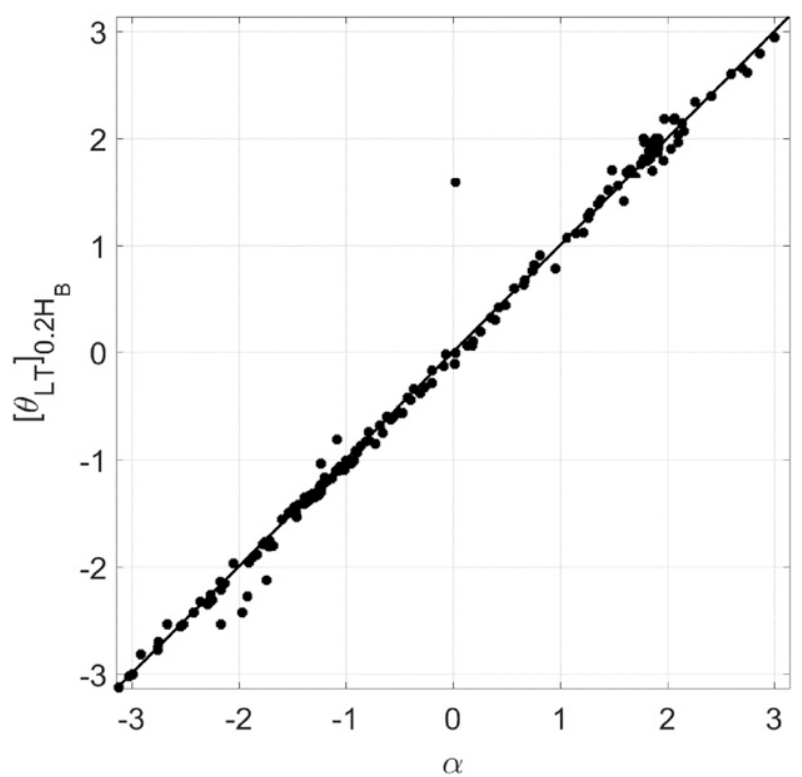

FIG. 14. The comparison of LT direction that is averaged within the upper $0.2 H_{B}$ to the direction of surface layer-averaged Lagrangian shear $\alpha$. LT direction $\theta_{\mathrm{LT}}$ is obtained at each depth level and then averaged throughout the upper $0.2 \mathrm{H}_{B}$, where the symbol [.] indicates a depth average. For $\alpha$, we average the Lagrangian shear first and then obtain the direction of such depth-averaged Lagrangian shear.

the important role of LT in inducing nonlocal transport in the upper OSBL, which needs to be considered for more comprehensive parameterizations of Reynolds stresses in larger-scale ocean models.

\section{Conclusions}

In this study, we have investigated the impact of windwave misalignment on LT directional variability and intensity that is characterized by the depth-averaged vertical velocity variance (VVV) under complex tropical cyclone wind and wave forcing. A tuned Holland wind model and the WAVEWATCH III wave model are used to build the TC wind and wave fields. These fields are then used to drive the upper ocean response to the TC that is simulated by a largeeddy simulation model based on filtered CraikLeibovich equations. The numerical experiments are conducted at multiple stations across the entire TC domain, which resolves the spatiotemporal variation of transient TC forcing.

We objectively identify LT direction at each depth based on its elongated structures, which are illustrated by autocorrelation of vertical velocity at different depth. In spite of the widely distributed wind-wave misalignments under the TC, LT direction is uniformly oriented to the wind in the upper $20 \%$ of the boundary layer depth $H_{B}$. This is because the Lagrangian shear, which determines LT direction, is aligned with the wind, and the Stokes drift shear, which predominantly contributes to the Lagrangian shear, is also along the wind. In addition, the alignment between the Stokes drift shear and the wind is due to the wind-driven waves, which feature relatively short wavelengths and small wind misalignment in the open ocean.

We also investigate the three-dimensional structure of dominant Langmuir cells (LCs), the orientation of which is important in understanding LT direction. The direction of dominant LCs is determined by the Lagrangian shear across the LC, which is approximately at a depth of maximum vertical velocity variance $H_{\left\langle w^{\prime 2}\right\rangle(\max )}$. In most regions under the TC, $H_{\left\langle w^{\prime 2}\right\rangle(\max )}$ is shallower than $0.2 H_{B}$, which is within the layer with
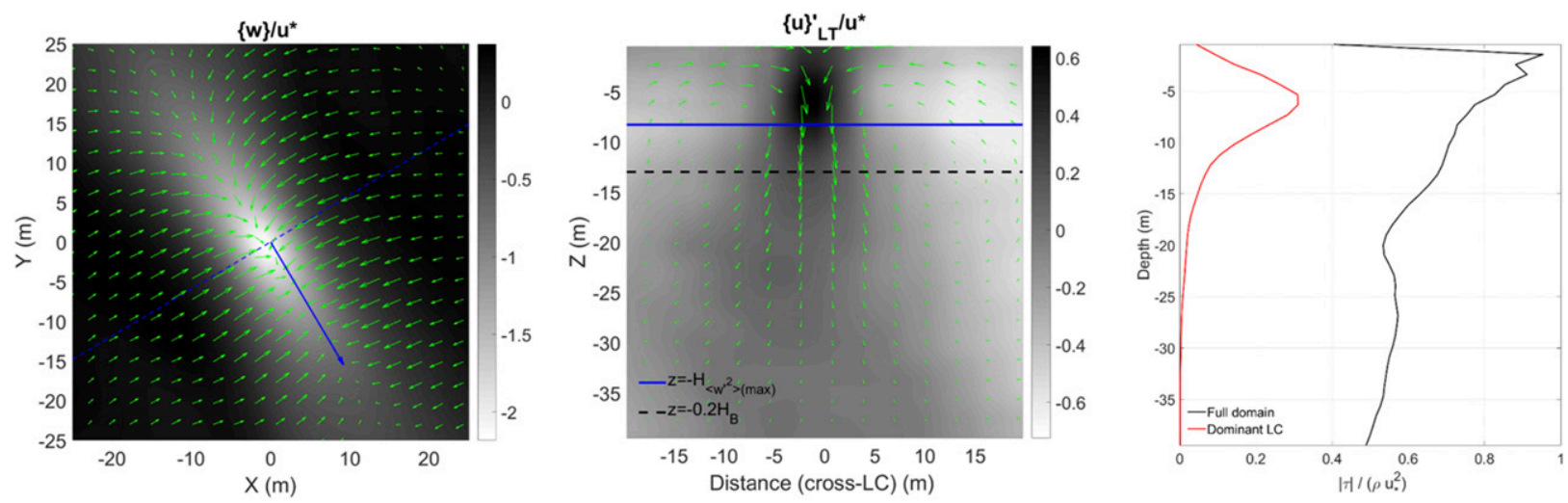

FIG. 15. (left) The map view of normalized vertical velocity at the depth $H_{\left\langle w^{\prime 2}\right\rangle(\max )}$. The thick blue arrow shows the direction of LT, and the blue dashed line represents the panel that is orthogonal to LT direction. The green arrows indicate the horizontal velocity vectors near the surface. (center) Normalized horizontal velocity anomalies that are projected into the panel normal to LT direction $\left(\{v\}_{\mathrm{LT}}^{\prime}\right)$ and the along LT part $\left(\{u\}_{\mathrm{LT}}^{\prime}\right)$. The green arrows are velocity vectors projected to the same panel normal to LT direction. The blue solid line indicates the depth of $H_{\left\langle w^{\prime 2}\right\rangle(\max )}$, and the black dashed line is the depth of $0.2 H_{B}$. (right) The comparison of Reynolds stress due to dominant Langmuir cells (solid red line) to the total Reynolds stress due to all turbulent motions (solid black line). 


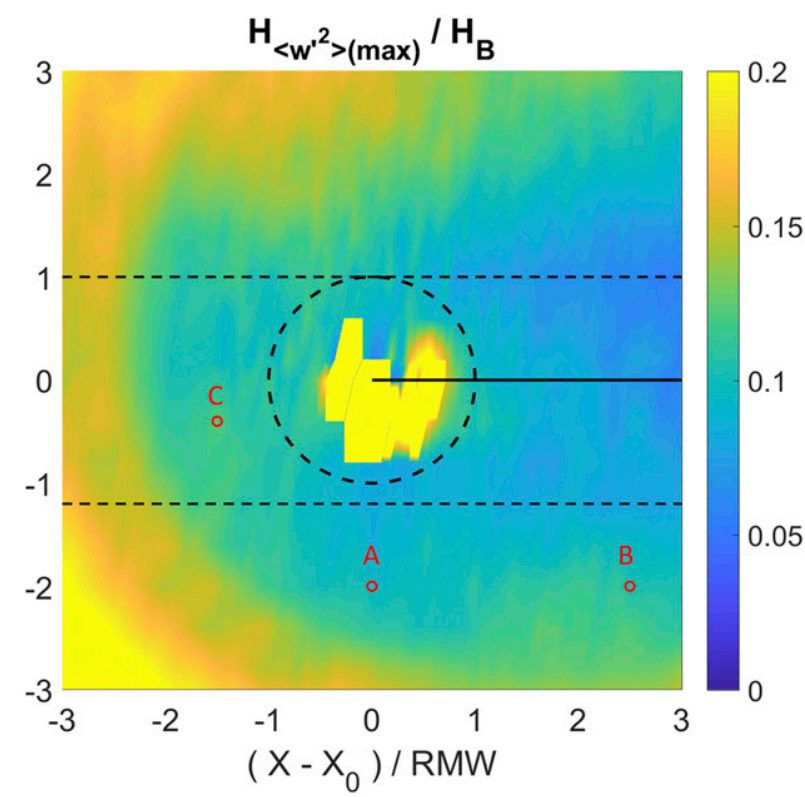

FIG. 16. The ratio of the depth of the maximum vertical velocity variance $H_{\left\langle w^{\prime 2}\right\rangle(\max )}$ to the boundary layer depth $H_{B}$. The color is saturated to bright yellow at values which are greater than 0.2 . Other line styles are the same as in Fig. 6.

uniformly wind-aligned Lagrangian shear. As a result, the Lagrangian shear at $H_{\left\langle w^{\prime 2}\right\rangle(\max )}$ is also aligned with the wind, suggesting wind-aligned dominant LCs. Thus, it is reasonable to use the Lagrangian shear at $H_{\left\langle w^{\prime 2}\right\rangle(\max )}$ to infer LT direction for our study. This shear is found similar to the depth-averaged Lagrangian shear.

Though the wind-wave misalignment does not impact LT direction, it plays a critical role in affecting LT intensity. Our results reveal that the total Stokes drift shear production $P_{S}$ is not consistent with the reduced LT intensity. For instance, we find stations with approximately the same depth-integrated $P_{S}$ but different VVV. This is because most $P_{S}$ concentrates in a surface layer, where Reynolds stress is nearly constant. As a result, the depth-integrated $P_{S}$ only depends on the dot product of the wind stress vector and surface Stokes drift vector. Given that surface Stokes drift is mostly controlled by windforced short waves and is approximately along the wind, the total $P_{S}$ is independent of the wind-wave misalignments, and thereby cannot be used to indicate the impact of the wind-wave misalignment on LT intensity.

To understand the layer with substantial stress, we assess the profile of Reynolds stress by scaling the momentum balance equation. The primary momentum balance varies with transient $\mathrm{TC}$ wind forcing and is indicated by a ratio of a time scale associated with the mean current change rate to the time scale of inertial oscillation. We find that the characteristics of the stress profile change with different types of momentum balances. For instance, when the currents are strongly forced by wind, the Reynolds stress is aligned with the wind throughout the $H_{B}$ and approximately linearly decays with depth. When the Coriolis force balances the divergence of the Reynolds stress, however, the stress profile satisfies the Ekman spiral structure, which rotates and decays with depth. Based on the scaling analysis of the stress profile, we obtain a conservative estimation of the thickness of the layer with substantial stress, which is deeper than the layer with predominant $P_{S}$.

We employ the same turbulent kinetic energy (TKE) model as in Wang et al. (2018) to explore the mechanism behind the reduced LT intensity by the wind-wave misalignment, which assumes a balance between $P_{S}$ and turbulent dissipation. Considering the wind-aligned, nearly constant Reynolds stress layer with predominant $P_{S}$, the derivation of the TKE model reveals that it is the windprojected Stokes drift that determines LT intensity. As such, the effect of the wind-wave misalignment on LT intensity can be assessed through the misalignment between wind and a depth-averaged Stokes drift, which is widely distributed over the TC domain and shows great spatiotemporal variation. In summary, our study emphasizes that LT intensity and direction are controlled by different wave parameters, which exhibit different responses to the wind-wave misalignment.

Acknowledgments. We acknowledge the support of NSF Grants OCE-1130678, OCE-1634578, and OCE1352422 for funding this work. We also thank the highperformance computing resources as well as Information Technologies support from NCAR Large computation allocation grant, sponsored by National Science Foundation and University of Delaware's high-performance computers Mills and Farber. We thank two anonymous reviewers for constructive comments that have improved our manuscript.

\section{REFERENCES}

Craig, P. D., and M. L. Banner, 1994: Modeling wave-enhanced turbulence in the ocean surface layer. J. Phys. Oceanogr., 24, 2546-2559, https://doi.org/10.1175/1520-0485(1994)024<2546: MWETIT $>2.0 . \mathrm{CO} ; 2$.

Craik, A. D. D., and S. Leibovich, 1976: A rational model for Langmuir circulations. J. Fluid Mech., 73, 401-426, https:// doi.org/10.1017/S0022112076001420.

Fan, Y., I. Ginis, T. Hara, C. W. Wright, and E. J. Walsh, 2009: Numerical simulations and observations of surface wave fields under an extreme tropical cyclone. J. Phys. Oceanogr., 39, 2097-2116, https://doi.org/10.1175/2009JPO4224.1.

Gnanadesikan, A., and R. A. Weller, 1995: Structure and instability of the Ekman spiral in the presence of surface gravity waves. J. Phys. Oceanogr., 25, 3148-3171, https://doi.org/10.1175/ 1520-0485(1995)025<3148:SAIOTE > 2.0.CO;2.

Grant, A. L. M., and S. E. Belcher, 2009: Characteristics of Langmuir turbulence in the ocean mixed layer. J. Phys. Oceanogr., 39, 1871-1887, https://doi.org/10.1175/2009JPO4119.1. 
Harcourt, R. R., and E. A. D'Asaro, 2008: Large-eddy simulation of Langmuir turbulence in pure wind seas. J. Phys. Oceanogr., 38, 1542-1562, https://doi.org/10.1175/2007JPO3842.1.

Holland, G. J., 1980: An analytical model of the wind and pressure profiles in hurricanes. Mon. Wea. Rev., 108, 1212-1218, https:// doi.org/10.1175/1520-0493(1980)108<1212:AAMOTW>2.0.CO;2.

- 2008: A revised hurricane pressure-wind model. Mon. Wea. Rev., 136, 3432-3445, https://doi.org/10.1175/2008MWR2395.1.

Holm, D. D., 1996: The ideal Craik-Leibovich equations. Physica D, 98, 415-441, https://doi.org/10.1016/0167-2789(96)00105-4.

Kenyon, K. E., 1969: Stokes drift for random gravity waves. J. Geophys. Res., 74, 6991-6994, https://doi.org/10.1029/JC074i028p06991.

Kukulka, T., and K. Brunner, 2015: Passive buoyant tracers in the ocean surface boundary layer: 1 . Influence of equilibrium windwaves on vertical distributions. J. Geophys. Res. Oceans, 120, 3837-3858, https://doi.org/10.1002/2014JC010487.

, and R. R. Harcourt, 2017: Influence of Stokes drift decay scale on Langmuir turbulence. J. Phys. Oceanogr., 47, 16371656, https://doi.org/10.1175/JPO-D-16-0244.1.

- , A. J. Plueddemann, J. H. Trowbridge, and P. P. Sullivan, 2010: Rapid mixed layer deepening by the combination of Langmuir and shear instabilities: A case study. J. Phys. Oceanogr., 40, 2381-2400, https://doi.org/10.1175/2010JPO4403.1.

_ - — , and P. P. Sullivan, 2012: Nonlocal transport due to Langmuir circulation in a coastal ocean. J. Geophys. Res., 117, C12007, https://doi.org/10.1029/2012JC008340.

_, R. L. Jenkins, J. T. Kirby, F. Shi, and R. W. Scarborough, 2017: Surface wave dynamics in Delaware Bay and its adjacent coastal shelf. J. Geophys. Res. Oceans, 122, 8683-8706, https:// doi.org/10.1002/2017JC013370.

Leibovich, S., 1983: The form and dynamics of Langmuir circulations. Annu. Rev. Fluid Mech., 15, 391-427, https://doi.org/ 10.1146/annurev.fl.15.010183.002135.

Li, M., C. Garrett, and E. Skyllingstad, 2005: A regime diagram for classifying turbulent large eddies in the upper ocean. Deep-Sea Res. I, 52, 259-278, https://doi.org/10.1016/j.dsr.2004.09.004.

McWilliams, J. C., and P. P. Sullivan, 2000: Vertical mixing by Langmuir circulations. Spill Sci. Technol. Bull., 6, 225-237, https://doi.org/10.1016/S1353-2561(01)00041-X.

,$- \ldots$, and C.-H. Moeng, 1997: Langmuir turbulence in the ocean. J. Fluid Mech., 334, 1-30, https://doi.org/10.1017/ S0022112096004375.

— - E. Huckle, J.-H. Liang, and P. P. Sullivan, 2012: The wavy Ekman layer: Langmuir circulations, breaking waves, and Reynolds stress. J. Phys. Oceanogr., 42, 1793-1816, https:// doi.org/10.1175/JPO-D-12-07.1.

,-- J. Liang, and P. P. Sullivan, 2014: Langmuir turbulence in swell. J. Phys. Oceanogr., 44, 870-890, https://doi.org/ 10.1175/JPO-D-13-0122.1.

Noh, Y., H. S. Min, and S. Raasch, 2004: Large eddy simulation of the ocean mixed layer: The effects of wave breaking and Langmuir circulation. J. Phys. Oceanogr., 34, 720-735, https://doi.org/ 10.1175/1520-0485(2004)034<0720:LESOTO > 2.0.CO;2.

Polton, J. A., and S. E. Belcher, 2007: Langmuir turbulence and deeply penetrating jets in an unstratified mixed layer. J. Geophys. Res., 112, C09020, https://doi.org/10.1029/2007JC004205.

, Y.-D. Lenn, S. Elipot, T. K. Chereskin, and J. Sprintall, 2013: Can Drake Passage observations match Ekman's classic theory? J. Phys. Oceanogr., 43, 1733-1740, https://doi.org/10.1175/JPO-D-13-034.1.
Pope, S. B., 2010: Turbulent Flows. 1st ed. Cambridge University Press, 93 pp.

Portilla, J., F. J. Ocampo-Torres, and J. Monbaliu, 2009: Spectral partitioning and identification of wind sea and swell. J. Atmos. Oceanic Technol., 26, 107-122, https://doi.org/ 10.1175/2008JTECHO609.1.

Price, J. F., R. A. Weller, and R. R. Schudlich, 1987: Wind-driven ocean currents and Ekman transport. Science, 238, 1534-1538, https://doi.org/10.1126/science.238.4833.1534.

Rabe, T. J., T. Kukulka, I. Ginis, T. Hara, B. G. Reichl, E. A. D'Asaro, R. R. Harcourt, and P. P. Sullivan, 2015: Langmuir turbulence under Hurricane Gustav (2008). J. Phys. Oceanogr., 45, 657-677, https://doi.org/10.1175/JPO-D-14-0030.1.

Reichl, B. G., I. Ginis, T. Hara, B. Thomas, T. Kukulka, and D. Wang, 2016a: Impact of sea-state-dependent Langmuir turbulence on the ocean response to a tropical cyclone. Mon. Wea. Rev., 144, 4569-4590, https://doi.org/10.1175/MWR-D-16-0074.1.

—, D. Wang, T. Hara, I. Ginis, and T. Kukulka, 2016b: Langmuir turbulence parameterization in tropical cyclone conditions. J. Phys. Oceanogr., 46, 863-886, https://doi.org/10.1175/JPO-D-15-0106.1.

Sinha, N., E. T.-M. Andres, and C. M. Akan, 2015: Toward a K-profile parameterization of Langmuir turbulence in shallow coastal shelves. J. Phys. Oceanogr., 45, 2869-2895, https:// doi.org/10.1175/JPO-D-14-0158.1.

Skyllingstad, E. D., and D. W. Denbo, 1995: An ocean large-eddy simulation of Langmuir circulations and convection in the surface mixed layer. J. Geophys. Res., 100, 8501-8522, https:// doi.org/10.1029/94JC03202.

Smyth, W. D., E. D. Skyllingstad, G. B. Crawford, and H. Wijesekera, 2002: Nonlocal fluxes and Stokes drift effects in the K-profile parameterization. Ocean Dyn., 52, 104-115, https://doi.org/10.1007/s10236-002-0012-9.

Sullivan, P. P., J. C. McWilliams, and W. K. Melville, 2007: Surface gravity wave effects in the oceanic boundary layer: Large-eddy simulation with vortex force and stochastic breakers. J. Fluid Mech., 593, 405-452, https://doi.org/10.1017/S002211200700897X. , L. Romero, J. C. McWilliams, and W. K. Melville, 2012: Transient evolution of Langmuir turbulence in ocean boundary layers driven by hurricane winds and waves. J. Phys. Oceanogr., 42, 1959-1980, https://doi.org/10.1175/JPO-D-12-025.1.

Terray, E. A., M. A. Donelan, Y. C. Agrawal, W. M. Drennan, K. K. Kahma, A. J. Williams, P. A. Hwang, and S. A. Kitaigorodskii, 1996: Estimates of kinetic energy dissipation under breaking waves. J. Phys. Oceanogr., 26, 792-807, https://doi.org/10.1175/ 1520-0485(1996)026<0792:EOKEDU > 2.0.CO;2.

Tolman, H. L., 2009: User manual and system documentation of WAVEWATCH III version 3.14. NOAA/NWS/NCEP/MMAB Tech. Note 276, 194 pp., http://polar.ncep.noaa.gov/mmab/ papers/tn276/MMAB_276.pdf.

Van Roekel, L. P., B. Fox-Kemper, P. P. Sullivan, P. E. Hamlington, and S. R. Haney, 2012: The form and orientation of Langmuir cells for misaligned winds and waves. J. Geophys. Res., 117, C05001, https://doi.org/10.1029/2011JC007516.

Wang, D., T. Kukulka, B. G. Reichl, T. Hara, I. Ginis, and P. P. Sullivan, 2018: Interaction of Langmuir turbulence and inertial currents in the ocean surface boundary layer under tropical cyclones. J. Phys. Oceanogr., 48, 1921-1940, https:// doi.org/10.1175/JPO-D-17-0258.1. 